\title{
Bodenkundliche Untersuchungen im spät- und postglazialen Bereich des Hinteren Lauterbrunnentals (Berner Oberland, Schweiz): Bodenchemischer und -mineralogischer Vergleich zweier Podsole auf unter- schiedlich alten Moränen
}

Markus Egli, Zürich

Aldo Mirabella, Firenze

\section{Bodenbildung und Problematik der zeitlichen Einordnung}

Der Verwitterungsgrad von Böden kann Auskunft über deren Alter und somit über die Genese der Landschaft geben. So einfach auch diese Aussage erscheinen mag, die Altersbestimmung und relative Zuordnung von Böden im alpinen Raum ist mit beträchtlichen Schwierigkeiten verbunden. Dies hat praktische sowie methodische Gründe. Um zumindest einen relativen Vergleich zwischen dem Verwitterungsgrad einzelner Böden erzielen zu können, müssen sich diese auf einem ähnlichen Gestein, in ähnlicher Topographie mit ähnlichem Klima entwickelt haben und zudem ein unterschiedliches Bodenalter aufweisen. Dieses Konzept lehnt sich sehr stark an die wegweisende Gleichung von JenNy $(1941,1980)$ an, nach welcher die Bodenbildung bzw. die Bodeneigenschaften als Funktion folgender Faktoren gesehen werden können

$$
\text { Soil property }=f(p t, c l, t p, t, o r g)
$$

mit $p t$ als Ausgangsmaterial, $c l$ als Klima, $t p$ als Topographie, $t$ als Bodenalter und org als Organismen. Bodensequenzen (wie bsp. Chronosequenzen) ergeben sich dann, wenn einer dieser Faktoren variabel (z.B. das Bodenalter) ist und die restlichen von untergeordneter Bedeutung oder konstant sind. Diese Voraussetzungen schränken die Auswahl an möglichen Standorten sehr stark ein. Hinzu kommt, dass die Böden im alpinen Raum sehr wenig untersucht (THEURILLAT et al.1998) sind, da sie häufig schlecht erreichbar sind und meist einen hohen Skelettanteil (Durchmesser $>2 \mathrm{~mm}$ ) aufweisen, welcher die Analyse und alle weiteren Berechnungen stark erschwert. Eine Bestimmung der Verwitterungsrate von chemischen Substanzen in alpinen Böden wird aus diesen Gründen praktisch nie unternommen. Des weiteren finden sich auch nur ganz selten Verknüpfungen der Bodenmineralogie mit dem unterschiedlichen Entwicklungsgrad der Böden (EgGenberger 1995, Righi et al. 1999). Neben dem Bodenchemismus ist auch die Mineralogie von eminenter Bedeutung, da während der Bodenbildung Mineralien einerseits aufgelöst und andererseits umge- formt werden. Aufgrund der mineralogischen Zusammensetzung können Rückschlüsse auf den Verwitterungsgrad eines Bodens gezogen werden. Meistens werden, wenn überhaupt, relative Altersangaben über die Konzentrationsverhältnisse bestimmter Elemente gemacht (FITZE 1982).

Die Berechnung von Elementverlusten innerhalb von Bodenprofilen gab für verschiedene Untersuchungsgebiete ausserhalb des Alpenraums zum Teil recht eindrückliche Chronosequenzen und somit Differenzierungsmöglichkeiten von Böden unterschiedlicher Entwicklung (JerSAK et al. 1995, Olsson \& Melkerud 2000). Die entsprechenden Berechnungsgrundlagen wurden aber erst kürzlich revidiert (EGLI \& FITZE 2000), so dass diverse, früher erzielte Ergebnisse zum Teil neu interpretiert werden müssen. Ein weiterer Ansatzpunkt bietet die Berechnung von Verwitterungsindizes (Reiche 1943, Vogel 1975, Kronberg \& NesBITT 1981, HaRNoIs 1988). In einigen Untersuchungsgebieten konnten mit dieser Methodik gute Ergebnisse erzielt werden (B̈̈UMLER et al. 1991, BÄUMLER et al. 1996).

Im vorliegenden Artikel versuchen wir zwei verschieden alte Podsole in einem alpinen Untersuchungsgebiet mit bodenchemischen und -mineralogischen Methoden zu unterscheiden und den entsprechenden Bezug zur Genese bzw. Gletschergeschichte herzustellen.

\section{Untersuchungsgebiet und Beprobungsstandorte}

Einen detaillierten Überblick über die geologische und geomorphologische Situation sowie über die entsprechende Gletschergeschichte im Untersuchungsgebiet Schmadri (Hinteres Lauterbrunnental, Schweiz) gibt WIPF (2001). Die Geologie variiert innerhalb des Untersuchungsgebietes relativ stark (WIPF 2001). Über dem Lauterbrunner Kristallin (Aarmassiv) schliessen sich autochthone Sedimente aus der Trias (Sandsteine mit Einlagerungen eines grünlichen Tonschiefers sowie ein Dolomitband), dem Jura (Eisenoolith, Malmkalk u.a.) und dem Tertiär (Mürrenbreccie u.a.). Die Untersuchungsstandorte (Bodenprofile) befinden sich auf Moränen bestehend aus granitischem Material (Lauterbrunner Kristallin, Aarmassiv).

Das Untersuchungsgebiet wird durch ein rauhes Klima mit einer jährlichen Mitteltemperatur von ca. 0 bis $0.5^{\circ} \mathrm{C}$ und Niederschlagshöhen im Bereich von ca. 


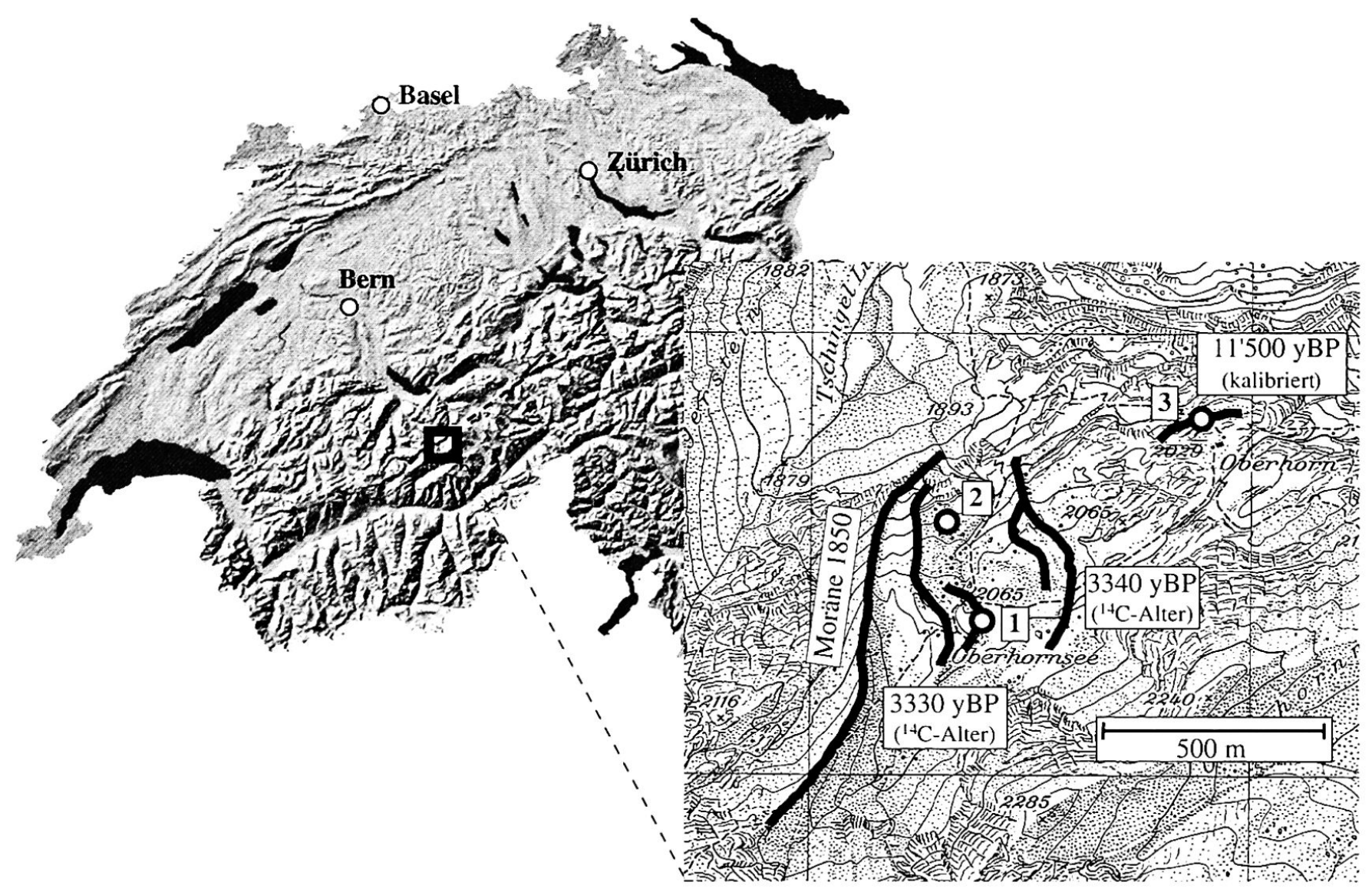

Abb. 1: Lage des Untersuchungsgebietes (Schmadri) und Bodenprofilstandorte (Kreise)

Location of the study area (Schmadri) and investigated soil profiles

Localisation de la région étudiée (Schmadri) et des profiles pédologiques (cercles)

reproduziert mit Bewilligumg vom 7.6.01 des VermessungSAmTES DES Kantons Bern

$2000 \mathrm{~mm}$ geprägt. Die untersuchten Böden befinden sich in einem geographisch sehr engen Bereich und auf einer Höhe von 2000 bis 2050 m ü.M. Aufgrund der zum Teil wechselnden Geologie konnten nur drei Standorte mit der gleichen Petrographie untersucht werden (Abb. 1). Die Bodentypen an den Standorten 1 und 2 (Abb. 1) waren nahezu identisch hinsichtlich der Horizontabfolge, der Bodenmächtigkeit und auch dem Alter. Deshalb wurde von diesen beiden nur Standort 1 intensiver chemisch und mineralogisch untersucht. Aufgrund des bedeutend jüngeren Bodenalters (ca. 3'500 Jahre) gegenüber Standort 3 (ca. 11'500 Jahre) erhofften wir uns interessante Einblicke in die Prozesse der Bodenbildung. Die Alpengletscher haben in den letzten rund $12^{\prime} 000$ Jahren mehr oder weniger innerhalb des Moränenkranzes von 1850 geschwankt. Dies weist somit auf relativ stabile Klimaverhältnisse innerhalb dieser Periode hin (Burga \& Perret 1998, Keller 1994). Das heisst, dass die beobachteten Unterschiede an Bodeneigenschaften vor allem ein Produkt des Faktors Zeit sind.

\section{Methoden}

\subsection{Datierung}

Die Datierung der einzelnen hier beschriebenen Standorte erfolgte über die ${ }^{14} \mathrm{C}$-Methode. Die postglazialen Moränenstandorte befanden sich über fossilen Böden. Die Ermittlung des ${ }^{14} \mathrm{C}$-Alters des fossilen Humus bzw. der organischen Restsubstanz (3'330 und 3'340 yBP bzw. ein kalibriertes Alter von ca. 3'500 Jahren, MaISCH 2000) erlaubte somit eine genaue Bestimmung der darüber abgelagerten Moräne. In unmittelbarer Nähe der spätglazialen Moräne befindet sich ein kleines Moor. Die Bestimmung des ${ }^{14} \mathrm{C}$-Alters von organischen Pflanzenresten (Gyttia) der Moorbasis (10’390 yBP) gibt einen relativen klaren Hinweis über den Zeitpunkt des Gletscherrückzugs bzw. den Beginn der Bodenbildung (vor ca. 11'500 Kalender-Jahren, MAISCH 2000).

\subsection{Bodenchemie und -physik}

Um den Gesamtchemismus des Bodens erfassen zu können wurde sowohl die Fraktion $<2 \mathrm{~mm}$ als auch 
der Skelettgehalt (Fraktion $>2 \mathrm{~mm}$ ) untersucht. Die Bestimmung der Gesamtgehalte an $\mathrm{Ca}, \mathrm{Mg}, \mathrm{K}, \mathrm{Na}, \mathrm{Fe}$, $\mathrm{Al}, \mathrm{Mn}, \mathrm{Si}$, Ti und $\mathrm{Zr}$ erfolgte mit einem Totalaufschluss der getrockneten Proben (FITZE et al.2000). Die Analytik wurde mit Atomabsorptions-Spektrometrie (Flammentechnik und wo nötig mit Graphitrohr; Perkin Elmer,AAnalyst 700) durchgeführt. Zusätzlich wurden die dithionit- und oxalat-extrahierbaren Anteile von $\mathrm{Fe}, \mathrm{Al}$ und $\mathrm{Si}$ gemessen. Der Gesamt-Stickstoff und -Kohlenstoff wurde über einen $\mathrm{C} / \mathrm{H} / \mathrm{N}$ Analyzer (Elementar Vario EL, elementar Analysensysteme $\mathrm{GmbH}$ ) ermittelt. Nachdem die Proben mit $\mathrm{H}_{2} \mathrm{O}_{2}(3 \%)$ vorbehandelt wurden, wurden die Korngrössen der gröberen Partikel $(2000-32 \mu \mathrm{m})$ mit dem Nassiebverfahren und der kleineren $(<32 \mu \mathrm{m})$ mit dem Röntgensedimentometer (SediGraph 5100) ermittelt. Die Bestimmung der Bodendichte erfolgte über die Entnahme von ungestörten Bodenproben mittels Bohrkernen (Mittelwert aus 5 Kernen) aus den unterschiedlichen Tiefenbereichen. Die entnommene Menge an Bodenmaterial entsprach pro Tiefenbereich $2-4 \mathrm{~kg}$. Über ein $2 \mathrm{~mm}$ Sieb wurde der Skelettanteil abgetrennt und dessen Proportion zur Gesamtmenge bestimmt.

\subsection{Bodenmineralogie}

Die mineralogische Zusammensetzung wurde an der Tonfraktion gemessen, da sich Veränderungen zuerst im Bereich von Kleinstpartikeln äussern. Nach der Zerstörung des organischen Materials mit verdünntem und $\mathrm{Na}$-Acetat gepuffertem $\mathrm{H}_{2} \mathrm{O}_{2}(\mathrm{pH} 5)$ wurde die Tonfraktion des Bodens mittels Dispergierung mit Calgon und Sedimentation im Wasser abgetrennt. Die Tonfraktion wurde mit $\mathrm{Mg}$ gesättigt, von Chloriden getrennt und gefriergetrocknet. Röntgendiffraktogramme wurden in einem ersten Schritt im Bereich von $2^{\circ}$ bis $65^{\circ}$ mit einem Philipps APD 1700 Diffraktometer $(\mathrm{Cu}-\mathrm{K} \alpha$ Strahlung; Labor für Tonmineralogie, ETHZ) erzeugt. Die erhaltenen Daten wurden mit dem Softwarepaket DMSNT auf mögliche, vorkommende Mineralien hin analysiert. Die weiteren Messungen wurden mit Tropfpräparaten durchgeführt. Die Analysen erfolgten mit einem 3-kW Rigaku D/MAX III C Diffraktometer mit einem horizontalen Goniometer und $\mathrm{Cu}-\mathrm{K} \alpha$ Strahlung am Istituto Sperimentale per lo Studio e la Difesa del Suolo (Firenze). Folgende Präparationstechniken wurden angewandt: $\mathrm{Mg}$-Sättigung, Bedampfung mit Ethylen Glykol, K-Sättigung mit anschliessendem Erhitzen während zwei Stunden bei $335^{\circ}$ und $550^{\circ} \mathrm{C}$. Die störenden Einflüsse von amorphem Fe und $\mathrm{Al}$ wurden vorgängig mit einer Oxalat Behandlung der Proben eliminiert. Die Herstellung desorientierter Proben erfolgte mit Hilfe von aufgerauhten Glas-Haltern. Diese Proben wurden schrittweise im Bereich von 58 bis $64^{\circ} 2 \theta$ gescant. Zusätzlich wurde ein $\mathrm{Na}$-Citrat Auszug (gemäss TAMURA 1958; mit geringfügigen Modifikationen) an der Tonfraktion vorgenommen, um Zwi-
schenschicht-Ionen wie $\mathrm{Al}$ und $\mathrm{Fe}$ zu lösen. Die Proben wurden während 24 Stunden ohne Erneuerung des Extraktionsmittels bei einer Temperatur von $135^{\circ} \mathrm{C}$ in einem Autoklaven aufgeschlossen. Die damit erhaltenen Röntgendiffraktogramme konnten mit den unbehandelten Proben verglichen werden. Die Kompaktion von 2:1 Schichtsilikaten wird nach der K Sättigung intensiviert, falls Zwischenschicht-Ionen vorhanden sind. HIS (hydroxy-interlayered smectites) werden durch einen prägnanteren Peak bei $1.65 \mathrm{~nm}$ nach Behandlung mit Ethylen Glykol nachgewiesen.

Die digitalisierten Röntgendaten wurden geglättet und hinsichtlich dem Lorentz und Polarisationsfaktor korrigiert (Moore \& Reynolds 1997). Die Peak und Profilanalyse erfolgte mit dem Programm Origin PFM ${ }^{\mathrm{TM}}$ unter Anwendung des Pearson VII Algorhythmus nach erfolgter Glättung der Daten mit einer Fourier Transform Funktion. Dadurch lassen sich (a) teilweise überlagernde Peaks trennen, (b) die Position der Peaks genau lokalisieren und (c) die einzelnen Flächenanteile bestimmen (Mirabella et al. 1992, Hardy et al. 1999). Die Proben wurden zusätzlich mit Infrarot untersucht. Infrarotspektren (IR) der Proben wurden mit einem Perkin-Elmer 880 Spektrometer gemessen. Folgende Spektren wurden aufgenommen: 4000 bis $400 \mathrm{~cm}^{-1}$ an Pastillen mit $1 \mathrm{mg}$ Probe und 250 mg KBr sowie 4000 bis $3000 \mathrm{~cm}^{-1}$ an denselben Pastillen, jedoch bei $150^{\circ} \mathrm{C}$ geheizt.

Die Berechnung der normativen Mineralzusammensetzung basiert auf der Gesamtzusammensetzung des Bodens. Der Gehalt an Fe-Oxiden und -Hydroxiden wurde über den Dithionit-Auszug ermittelt und der Anteil an Imogolit-ähnlichen Mineralien gemäss PARFITT \& HeNmi (1982) berechnet.

\section{Ergebnisse und Diskussion}

\subsection{Allgemeine Bodeneigenschaften}

Einige grundlegende Eigenschaften der untersuchten Böden sind in Tab. 1 dargestellt. Beide Böden weisen typische Charakteristika von Podsolen auf: tiefe $\mathrm{pH}$ Werte im Oberboden sowie Verlagerung und Einwaschung von $\mathrm{Fe}, \mathrm{Al}$ und organischer Materie. Die Böden kennzeichnen sich im $\mathrm{C}$-Horizont durch einen relativ hohen Skelettgehalt aus. Mit zunehmender Verwitterung wird im allgemeinen die Grösse der Partikel verkleinert, was sich auch in einer entsprechenden Veränderung der Korngrösse mit zunehmender Nähe zur Erdoberfläche widerspiegelt. Moränen sind in ihrem Aufbau (im speziellen hinsichtlich der Korngrösse) häufig nicht sehr homogen. Den Wechsel im Skelettgehalt bzw. in der Korngrösse erklären wir einerseits durch diese Inhomogenität und andererseits durch Verwitterungsprozesse. Ein gewisser äolischer Einfluss kann nicht mit letzter Sicherheit ausgeschlossen werden. Wir müssen aber aufgrund des Boden- 


\begin{tabular}{|c|c|c|c|c|c|c|c|c|c|c|c|c|c|}
\hline $\begin{array}{l}\text { Standort } \\
\text { Horizontbereich } \\
\text { Tiefe }(\mathrm{cm})\end{array}$ & $\begin{array}{l}\text { Boden- } \\
\text { horizont }\end{array}$ & $\begin{array}{l}\text { Beprobungs- } \\
\text { tiefe }(\mathrm{cm})\end{array}$ & $\underset{\%^{3)}}{\text { Skelett }}$ & $\begin{array}{l}\text { Dichte } \\
\mathrm{g} \mathrm{cm}^{-3}\end{array}$ & $\left(\mathrm{CaCl}_{2}\right)$ & $\underset{\%}{\text { org. } C}$ & $\underset{\%}{\operatorname{org} . \mathrm{N}}$ & $\begin{array}{l}\mathrm{Al}_{\mathrm{d}}^{1 \mathrm{1}} \\
\mathrm{mg} / \mathrm{kg}\end{array}$ & $\begin{array}{l}\mathrm{Fe}_{\mathrm{d}}^{1)} \\
\mathrm{mg} / \mathrm{kg}\end{array}$ & $\begin{array}{l}\mathrm{Si}_{\circ}^{2)} \\
\mathrm{mg} / \mathrm{kg}\end{array}$ & $\operatorname{Sand}_{\%}^{5}$ & $\begin{array}{l}\mathrm{Silt}^{5} \\
\%\end{array}$ & $\begin{array}{l}\text { Ton } \\
\%^{4)}\end{array}$ \\
\hline \multicolumn{14}{|c|}{ 3'500 Jahre alter Haplic Podzol** } \\
\hline $0-8$ & $\mathrm{O}$ & $3-8$ & 0 & 0.57 & 3.5 & 14.28 & 0.87 & 1655 & 3580 & 17 & & & \\
\hline $8-15$ & $\mathrm{E}$ & $9-14$ & 25 & 0.77 & 3.7 & 6.91 & 0.32 & 3544 & 9810 & 17 & 53.8 & 30.1 & 16.1 \\
\hline $15-22$ & Bhs & $16-21$ & 27 & 1.36 & 3.8 & 11.26 & 0.44 & 7740 & 13080 & 52 & 51.3 & 32 & 16.7 \\
\hline $22-60$ & Bws & $28-38$ & 72 & 1.76 & 4.3 & 4.55 & 0.17 & 12920 & 20780 & 1725 & & & \\
\hline$>60$ & C & $75-85$ & 79 & 1.89 & 4.7 & 0.6 & 0.01 & 2408 & 2790 & 376 & 78 & 17 & 5 \\
\hline \multicolumn{14}{|c|}{ 11'500 Jahre alter Haplic Podzol } \\
\hline $0-10$ & O & $3-10$ & 0 & 0.39 & 3.9 & 38.33 & 1.8 & 2713 & 4590 & n.n.* & & & \\
\hline $10-16$ & E & $10-16$ & 7 & 1.09 & 3.7 & 2.23 & 0.08 & 766 & 1830 & n.n. & 54.4 & 32.6 & 13 \\
\hline $16-24$ & Bhs & $16-22$ & 23 & 1.01 & 3.8 & 11.31 & 0.48 & 10910 & 46230 & 93 & 49.1 & 30.7 & 18.7 \\
\hline $24-55$ & Bs & $24-34$ & 25 & 0.94 & 4.1 & 4.26 & 0.15 & 23620 & 18300 & 1104 & & & \\
\hline $55-105$ & Bw & $55-65$ & 26 & 1.54 & 4.5 & 0.95 & 0.02 & 4373 & 7830 & 851 & 73.7 & 21.3 & 5 \\
\hline$>105$ & $\mathrm{C}$ & 105 & 42 & 1.70 & 4.7 & 0.22 & 0 & 1645 & 4840 & 316 & 82.1 & 13 & 4.9 \\
\hline
\end{tabular}

"Dithionit-extrahierbare Fraktion

"Oxalat-extrahierbare Fraktion

3) Prozentualer Gewichtsanteil an der gesamten Masse

- Dichte des gesamten Materials inkl. Hohlräume (= scheinbare Dichte)

- n.n. = nicht nachweisbar

4) Prozentualer Anteil der Feinerde (<2 mm)

. Nomenklatur gemäss FAO-UNESCO

s) Sand $(0.062-2.0 \mathrm{~mm})$. Silt $(0.062-0.002 \mathrm{~mm})$, Ton $(<0.002 \mathrm{~mm})$

Tab. 1: Charakteristische Eigenschaften der untersuchten Böden

Characteristics of the investigated soils

Propriétés caractéristiques des sols étudiés

chemismus und der Bodenmineralogie (kein erhöhter Chlorit-Gehalt im Oberboden, kein Epidot, kein Dolomit) davon ausgehen, dass dieser Einfluss nicht sonderlich gross gewesen sein kann.

\subsection{Bodenparameter als Funktion der Zeit}

Dithionit-extrahierbare Al- und Fe-Formen:

Der Dithionit-Auszug löst verschiedene $\mathrm{Al}$ - und $\mathrm{Fe}$ Formen im Boden. Für Al werden folgende erwähnt (Wada 1977, Shoji \& FuJiwara 1984, BorgGaARd 1988, DAHLGREN \& UGOLINI 1991): organisches Al, nicht kristalline und kristalline Oxihydroxide, etwas $\mathrm{Al}$ in den Zwischenschichten und Allophane. Beim Fe werden vor allem nicht kristalline und kristalline $\mathrm{Fe}$-Oxihydroxide und zu einem gewissen Anteil auch Fe-haltige Silikate gelöst (z.B. Nontronit; BorggaArd 1988). Fitze (1982) zeigt, dass das $\mathrm{Al}_{\mathrm{d}} / \mathrm{Fe}_{\mathrm{d}}$ Verhältnis im B-Horizont ein guter Indikator für das Alter von Böden im alpinen Raum darstellt. Dieses Verhältnis ist, ergänzt mit weiteren Daten von Fitze (1982) sowie EgLl et al. (2001) aus ähnlichen Untersuchungsgebieten, in Abb. 2 dargestellt. Daraus ist ersichtlich, dass sich die beiden Podsole chronologisch tatsächlich auch gut einordnen lassen.

Das Verhältnis $\frac{\left[\mathrm{Al}_{t} \mathrm{Fe}_{d}\right]}{\left[\mathrm{Al}_{d} \mathrm{Fe}\right]}$ berücksichtigt die Gesamt-

produktion an dithionit-extrahierbarem $\mathrm{Fe}$ und $\mathrm{Al}$ als Verhältnis zum Totalgehalt. Wird dieses Verhältnis aufgrund der mittleren Konzentrationen $\operatorname{der} \mathrm{A}+\mathrm{E}+\mathrm{B}$ Horizonte gegen die Zeitdauer der Bodenentwicklung aufgetragen, so ist ein enger Zusammenhang ersichtlich. Diese sehr schön ausgeprägte Chronofunktion zeigt, dass vor allem in den ersten 2'000 bis 3'000 Jahren eine schnelle Abnahme des Verhältnisses zu messen ist. Danach nähert sich dieses Verhältnis einer Asymptote. Das bedeutet wiederum, dass in unserem Fall die Böden, welche ein Alter von ca. 3'500 und 11 '500 Jahren aufweisen, mit dieser Methode nur knapp voneinander unterschieden werden können.

\section{Stickstoff:}

Die Akkumulationsrate an $\mathrm{N}$ versteht sich als Bilanz aus dem Input über die Produktion an organischer Materie und dem Output über Abbauprozesse. Die Möglichkeit einer zeitlichen Einordnung der Böden über die organische Substanz wird in der Literatur kontrovers diskutiert. Absolute Konzentrationen geben in den meisten Fällen keinen Hinweis auf den Reifegrad eines Bodens (FITZE 1982). Die gespeicherte Menge an organischer Substanz in einem Bodenprofil kann hingegen im Hinblick auf eine chronologische Differenzierung durchaus zu sinnvollen Resultaten führen (Lichter 1998, Liski et al. 1998). Die Chronofunktion der N-Akkumulationsrate (als Vertreter der organischen Substanz) ist besonders gut ausgeprägt (Abb. 3). Die erhaltenen Daten wurden mit denjenigen aus dem Untersuchungsgebiet Gletsch ergänzt (EGLI et al. 2001). Aufgrund des N-Gehaltes lassen sich die beiden Podsole zeitlich sehr gut voneinander trennen: Der jüngere Podsol hat eine deutlich niedrigere Gesamtmenge und weist somit auch tatsächlich einen niedrigeren Bodenentwicklungsgrad auf.

\section{Verwitterungsindizes:}

Um die chemische Verwitterung von Böden zu charakterisieren wurden in der Literatur diverse Verwit- 
a)

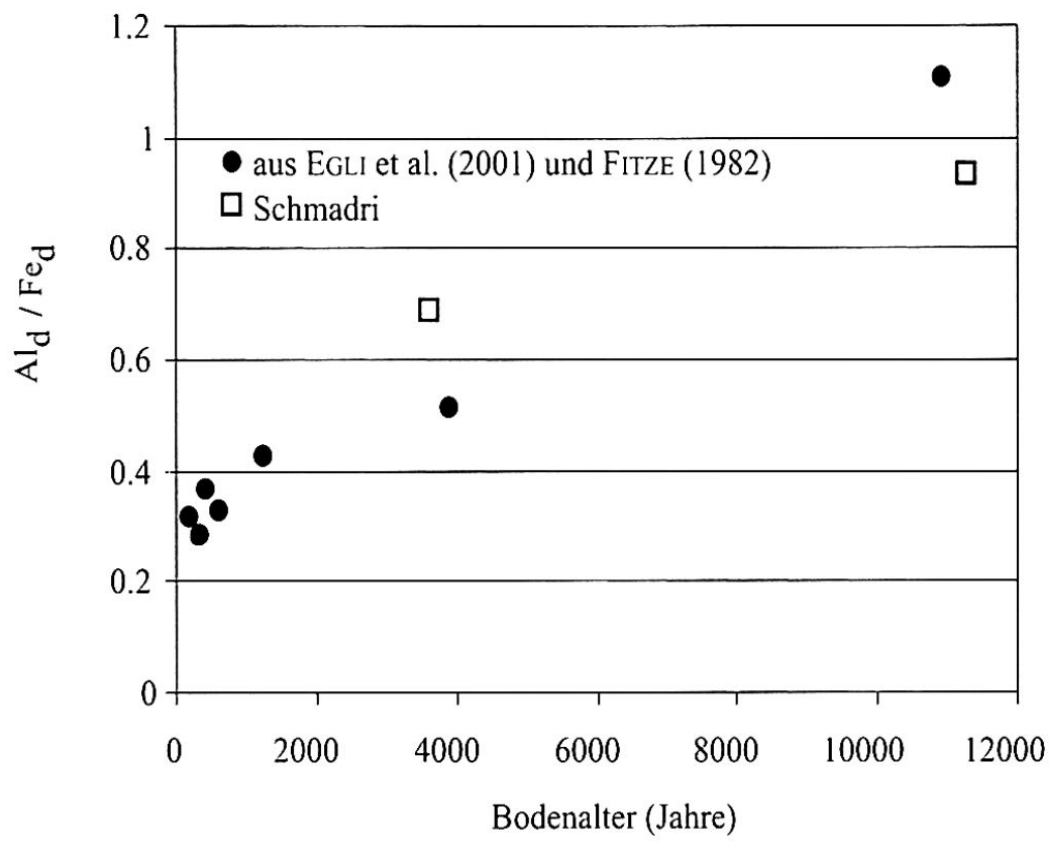

b)

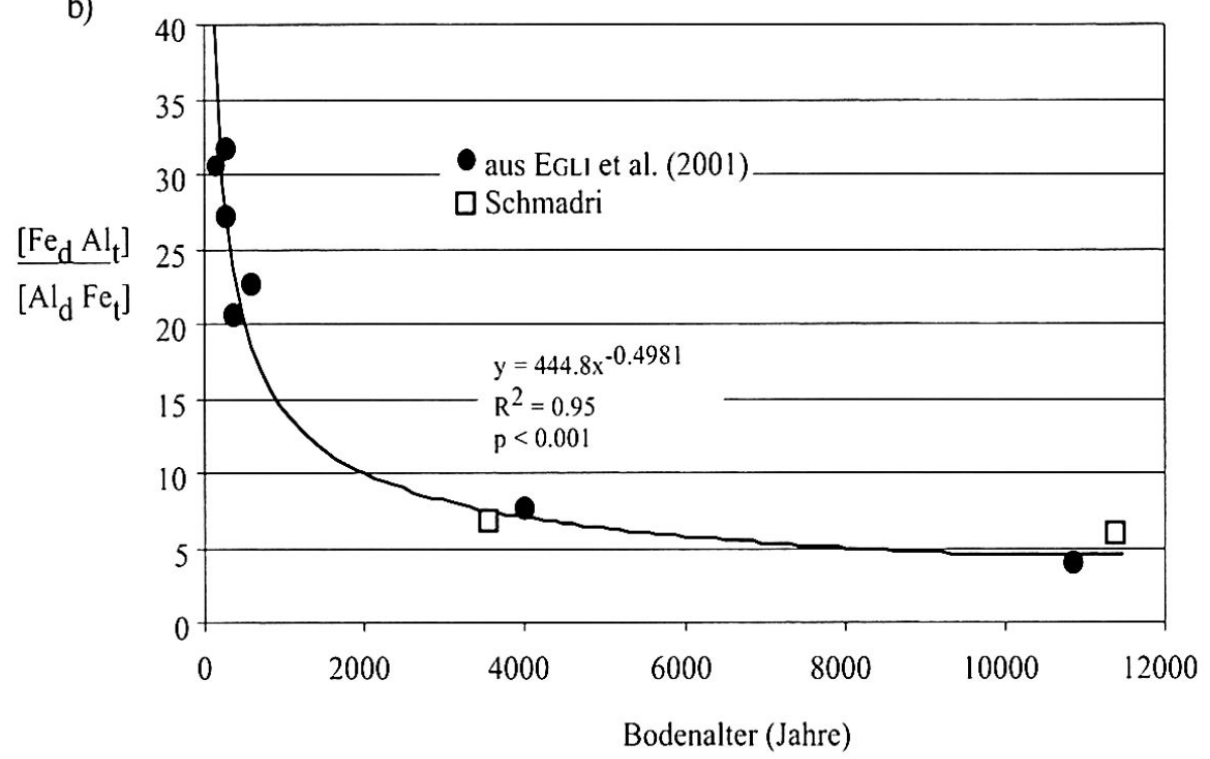

Abb. 2: Vergleich des a) $A l_{d} / F e_{d}$ Verhältnisses im B-Horizont und des b) mittleren $\left[A l_{t} F e_{d}\right] /\left[A l_{d} F e_{t}\right]$ Verhältnisses im A, E und B Horizont ( $d=$ dithionit-extrahierbare Fraktion, $t=$ Totalgehalt $)$ unterschiedlich entwickelter Böden mit der Zeit. Die Daten wurden mit Ergebnissen aus ähnlichen Untersuchungsgebieten (bezüglich Geologie, Klima und Topographie) ergänzt und zwar für a) mit Egli et al. (2001) und FITZE (1982) und b) mit EgLi et al. (2001).

Chronofunctions of a) the $A l_{d} / \mathrm{Fe}_{d}$ ratio ( $d$ = dithionite extractable fraction) in the $B$ horizon, $b$ ) the mean ratio $\left[A l_{t} F_{d}\right] /\left[A l_{d} F e_{d}\right]$ in the $A, E$ and $B$ horizon (with $t=$ total element content). The data have been completed with results obtained from similar investigation areas (with respect to the geology, climate and topography) for a) with EgLI et al. (2001) and FITZE (1982) and for b) with EGLI et al. (2001).

Comparaison des fonctions temporelles a) par rapport au quotient de $A l_{d} / F e_{d}$ (avec $d=$ extraction avec dithionite) et b) par rapport au quotient $\left[A l_{t} F e_{d}\right] /\left[A l_{d} F e_{t}\right]$ (avec $t=$ contenu total). Ces données ont été complétées par des résultats obtenus sur des sites similaires (par rapport à la géologie, au climat et à la topographie), c'est-à-dire pour a) avec EgLI et al. (2001) et FITZE (1982) et pour b) avec EgLI et al. (2001). 


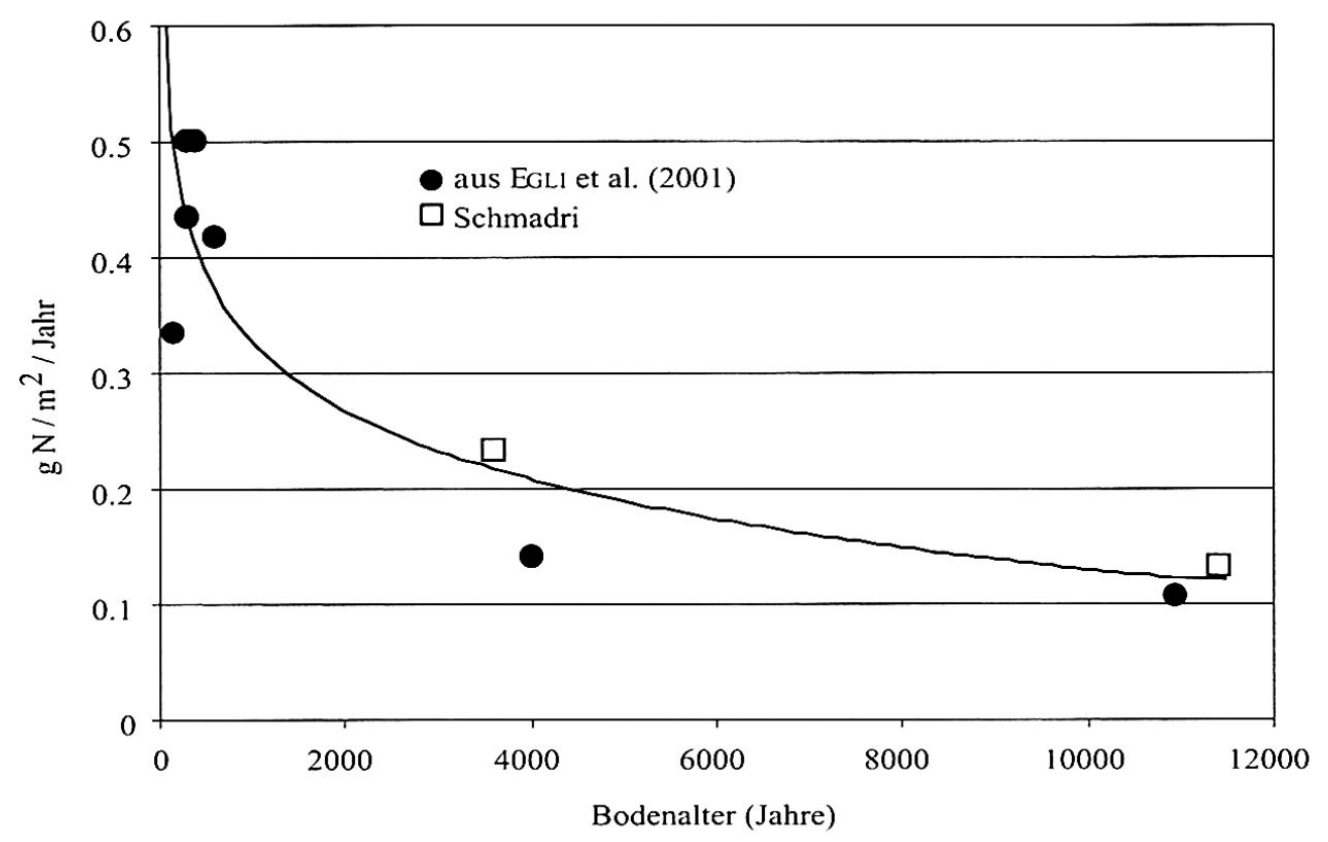

Abb. 3: Chronofunktion der Stickstoff-Akkumulationsrate im Boden

Accumulation rate of nitrogen as a function of the soil age

Fonction temporelle de l'accumulation de l'azote dans le sol

terungsindizes definiert. Das generelle Prinzip dieser Indizes ist mehr oder weniger immer ähnlich und basiert auf dem Verhältnis von basischen Kationen $(\mathrm{Ca}, \mathrm{K}, \mathrm{Na}, \mathrm{Mg}$ ) zu Al und/oder Si. Folgende Indizes wurden überprüft:

der MWPI-Index (auf molarer Basis) nach REICHE (1943)

MWPI $=\frac{100\left(\mathrm{CaO}+\mathrm{Na}_{2}\right.}{\mathrm{SiO}_{2}+\mathrm{Al}_{2} \mathrm{O}_{3}+\mathrm{Fe}_{2} \mathrm{O}_{3}+\mathrm{Na}_{2} \mathrm{O}+\mathrm{MgO}+\mathrm{K}_{2}+\mathrm{O}_{2} \mathrm{O}}$

der CIA und CIW-Index (Harnols 1988, Nesbitt \& Young 1989)

$$
\begin{aligned}
& \mathrm{CIA}=\frac{\mathrm{Al}_{2} \underline{\mathrm{O}}_{3}}{\mathrm{Al}_{2} \mathrm{O}_{3}+\mathrm{CaO}+\mathrm{Na}_{2} \mathrm{O}+\mathrm{K}_{2} \mathrm{O}} ; \\
& \mathrm{CIW}=\frac{\mathrm{Al}_{2} \underline{\mathrm{O}}_{3}}{\mathrm{Al}_{2} \mathrm{O}_{3}+\mathrm{CaO}+\mathrm{Na}_{2} \mathrm{O}}
\end{aligned}
$$

der Index A und B (auf molarer Basis) nach KRONBERG \& NESBITT (1981)

$$
\begin{aligned}
& \mathrm{A}=\frac{\mathrm{SiO}_{2} \pm \mathrm{CaO}+\mathrm{K}_{2} \mathrm{O}+\mathrm{Na}_{2} \mathrm{O}}{\mathrm{Al}_{2} \mathrm{O}_{3}+\mathrm{SiO}_{2}+\mathrm{CaO}+\mathrm{K}_{2} \mathrm{O}+\mathrm{Na}_{2} \mathrm{O}} ; \\
& \mathrm{B}=\frac{\mathrm{CaO}+\mathrm{K}_{2} \mathrm{O}+\mathrm{Na}_{2} \mathrm{O}}{\mathrm{Al}_{2} \mathrm{O}_{3}+\mathrm{CaO}+\mathrm{K}_{2} \mathrm{O}+\mathrm{Na}_{2} \mathrm{O}}
\end{aligned}
$$

Die Ergebnisse, welche mit diesen Verwitterungsindizes erzielt werden können, sind einander ähnlich. Stellvertretend für all diese Indizes werden diejeni- gen nach Kronberg \& Nesbitt (1981) dargestellt. Aus Abb. 4 kann eine generelle Tendenz im Verlaufe der Zeit zu niedrigeren Werten des Indexes B beobachtet werden. Die Streuung ist aber zu gross, als dass eine exaktere Eingliederung des Bodens aufgrund dieser Werte vorgenommen werden könnte. Auch die Gegenüberstellung des Indexes A mit B in Form eines cartesischen Koordinatensystems zeigt zwar eine generelle Tendenz (vgl. auch BÄUMLER et al. 1996), doch fehlt auch hier eine präzisere Möglichkeit nach einer Einordnung.

\subsection{Verwitterungsraten der Böden}

Die Tabellen 1 und 2 enthalten die geochemische Charakterisierung der Standorte. Diese Daten bieten die Grundlage zur Berechnung von Verwitterungsraten. Die Verwitterungsrate von Elementen wird üblicherweise über Konzentrationsprofile immobiler Elemente wie bspw. Ti oder $\mathrm{Zr}$ bestimmt. Mit Hilfe solcher immobilen Elemente werden Dehnungs- oder Kompaktionsfaktoren (im nachfolgenden als «Strain» definiert) bestimmt. Im Verlauf der Bodenentwicklung können sowohl positive wie auch negative Volumenänderungen eines Elementarvolumens im Boden erfolgen. Unter Berücksichtigung des Strains können elementspezifische Verluste oder Gewinne berechnet werden. Werden diese Gewinne oder Verluste über das gesamte Bodenprofil integriert und durch das Alter des Bodens dividiert, resultieren daraus die entsprechenden Verwitterungsraten. Das damit ver- 

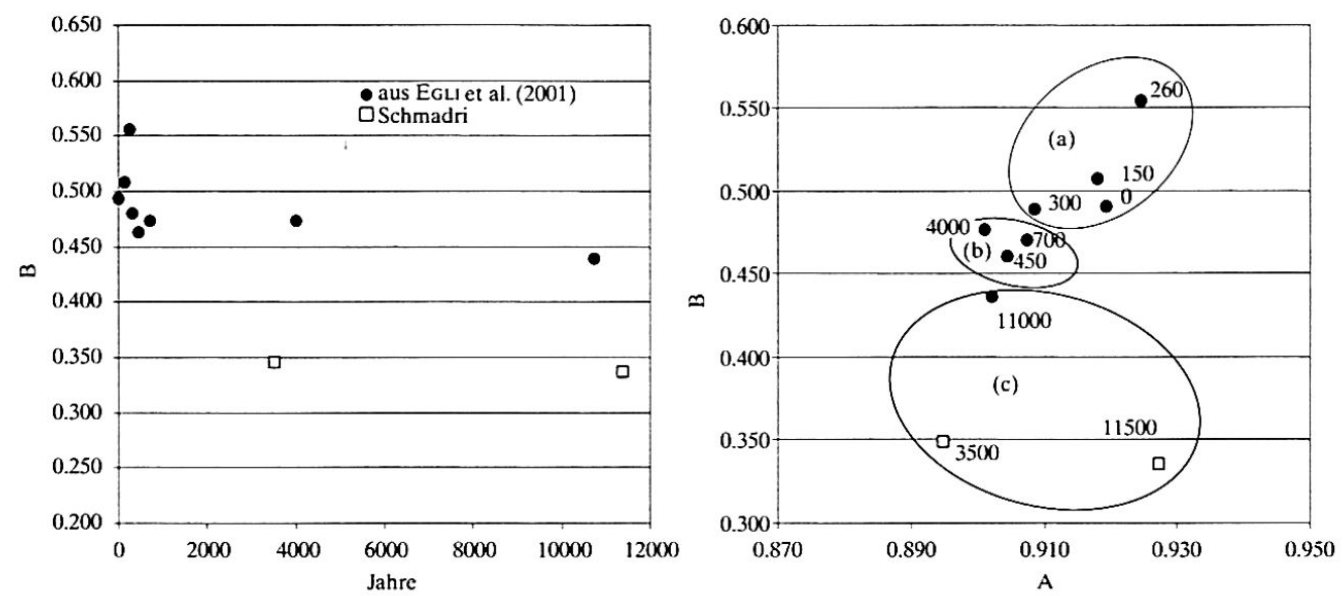

Abb. 4: Vergleich des Verwitterungsindexes mit dem Faktor Zeit und dem Index A. Die Daten wurden mit Resultaten aus EgLI et al. (2001) ergänzt. Die Indices sind für die Horizontbereiche mit der grössten Verwitterungsintensität berechnet. Gruppe (a) entspricht jungen, (b) mittleren und (c) älteren Böden.

Comparison of the weathering index $B$ with time and with index $A$. The data has been completed with results from EGLI et al. (2001). The indices are calculated for the horizons with the hihgest intensity of weathering. The group (a) represents young, (b) intermediate, and (c) older soils.

Comparaison de l'indice de la décomposition $B$ avec le facteur temps et avec l'indice A. Les données ont été complétées par les résultats d'EGLI et al. (2001). Les indices ont été calculés pour les horizons les plus décomposés. Le groupe (a) représente des sols jeunes, le groupe (b) des sols intermédiaires et le groupe (c) des sols plus vieux.

bundene mathematische Modell wurde von CHADwick et al. (1990) entwickelt und erst kürzlich von EGLI \& FITZE (2000) revidert. Volumenveränderungen während der Pedogenese berechnen sich über den Strain, $\varepsilon_{\mathrm{i}, \mathrm{w}}$ :

$$
\varepsilon_{i, w}=\frac{\Delta z_{w}}{\Delta z}-1
$$

mit $\Delta \mathrm{z}$ als Höhe $(\mathrm{m})$ eines entsprechenden Elementarvolumens des unverwitterten Ausgangsmaterials ' $\mathrm{p}$ ' («Protolit», Ausgangsgestein) und $\Delta z_{w}$ als Höhe (m) des Elementarvolumens im verwitterten Zustand ' $w$ '. Mit $\tau_{j, w}$ lassen sich die Massenbewegungen der Stoffe über die Grenze eines betrachteten Volumens hinaus beschreiben. $\tau_{j, w}$ steht für den relativen Verlust eines Stoffes gemessen an dessen Menge im unverwitterten Ausgangsmaterial.

$\left.\tau_{j, w}=\frac{\left(\rho_{w} C_{j, w}\right.}{\rho_{p} C_{j, p}}\left(\varepsilon_{i, w}+1\right)\right)-1$

$C_{j, p}$ ist die Konzentration des Elementes $j$ im Protolit $(\mathrm{kg} / \mathrm{t}), C_{j, w}$ die Konzentration des Elementes $j$ im Verwitterungsprodukt $(\mathrm{kg} / \mathrm{t})$ und $\rho_{p}$ und $\rho_{w}$ die scheinbare Dichte $\left(t / \mathrm{m}^{3}\right)$ des Protolits bzw. des Verwitterungsprodukts. Mit $n$ Bodenschichten lässt sich die Berechnung der Massenveränderungen $\bar{m}_{j, f l u x}\left(z_{w}\right)$ eines Elementes $j$ wie folgt darstellen (EGLI \& FITZE 2000):

$$
\bar{m}_{j, f l u x\left(z_{w}\right)}=\sum_{\mathrm{a}=1}^{\mathrm{n}} C_{j, p} \rho_{p}\left(\frac{1}{\varepsilon_{\mathrm{i} . \mathrm{w}}+1}\right) \tau_{j, w} \Delta z_{\mathrm{w}}
$$

Stark positive Strains werden vor allem in den obersten Bodenhorizonten beobachtet (Tab. 3). Die positiven Werte sind auf die Anwesenheit von organischer Substanz, welche eine geringere Dichte aufweist als die mineralischen Bestandteile, und das Durchdringen von Wurzeln, welche zu einer Lockerung des Materials geführt haben, zurückzuführen. Aus der Tiefenverteilung des Strains lässt sich ableiten, dass die Verwitterung in den meisten Fällen nicht isovolumetrisch verläuft. Die Werte für $\tau_{j, w}$ sind in Tabelle 3 ersichtlich. Negative Werte deuten auf Verluste hin (z.B. -0.77 heisst, dass $77 \%$ der ursprünglich vorhandenen Mengen ausgetragen worden sind). Die Gegenüberstellung der beiden Podsole zeigt deutlich auf, dass insbesondere die basischen Kationen unterschiedliche Verluste haben können. Der ältere Podsol weist speziell beim $\mathrm{Na}$ (als Vertreter des Hauptminerals Plagioklas) tiefgründigere und grössere Verluste auf (bis zu $80 \%$ des ursprünglichen Gehaltes) als der jüngere Podsol. Auch die Verluste der Ionen Ca, 


\begin{tabular}{|c|c|c|c|c|c|c|c|c|c|c|c|c|c|c|c|}
\hline $\begin{array}{l}\text { Standort } \\
\text { Horizontbereich } \\
\text { Tiefe }(\mathrm{cm})\end{array}$ & $\begin{array}{l}\text { Boden- } \\
\text { horizont }\end{array}$ & $\begin{array}{c}\text { Beprobungs- } \\
\text { tiefe }(\mathrm{cm})\end{array}$ & $\underset{\%}{\mathrm{SiO}_{2}}$ & $\underset{\%}{\mathrm{CaO}}$ & $\underset{\%}{\mathrm{MgO}}$ & $\underset{\%}{\mathrm{Na}_{2} \mathrm{O}}$ & $\underset{\%}{\mathrm{~K}_{2} \mathrm{O}}$ & $\underset{\%}{\mathrm{MnO}}$ & $\underset{\%}{\mathrm{Fe}_{2} \mathrm{O}_{3}}$ & $\underset{\%}{\mathrm{Al}_{2} \mathrm{O}_{3}}$ & $\underset{\%}{\mathrm{TiO}_{2}}$ & $\underset{\% 0}{\mathrm{ZrO}_{2}}$ & org. Substanz & $\begin{array}{l}\Sigma \\
\%\end{array}$ & $\begin{array}{c}\text { Rest* } \\
\%\end{array}$ \\
\hline $\begin{array}{r}\text { 3'500 Jahre alter H } \\
0-8 \\
8-15 \\
15-22 \\
22-60 \\
>60\end{array}$ & $\begin{array}{l}\text { Podzol }{ }^{* * *} \\
\text { O } \\
\text { E } \\
\text { Bhs } \\
\text { Bws } \\
\text { C }\end{array}$ & $\begin{array}{c}3-8 \\
9-14 \\
16-21 \\
28-38 \\
75-85\end{array}$ & $\begin{array}{l}38.7 \\
61.7 \\
61.3 \\
62.9 \\
66.5\end{array}$ & $\begin{array}{l}0.31 \\
0.22 \\
0.28 \\
0.35 \\
0.43\end{array}$ & $\begin{array}{l}0.55 \\
1.04 \\
1.14 \\
1.61 \\
1.73\end{array}$ & $\begin{array}{l}1.20 \\
1.84 \\
1.62 \\
2.37 \\
2.88\end{array}$ & $\begin{array}{l}2.21 \\
3.37 \\
3.03 \\
3.30 \\
3.63\end{array}$ & $\begin{array}{l}0.01 \\
0.03 \\
0.04 \\
0.08 \\
0.07\end{array}$ & $\begin{array}{l}1.36 \\
3.59 \\
4.50 \\
4.65 \\
4.48\end{array}$ & $\begin{array}{c}8.61 \\
13.37 \\
11.79 \\
15.37 \\
15.02\end{array}$ & $\begin{array}{l}1.10 \\
1.17 \\
1.00 \\
0.81 \\
0.85\end{array}$ & $\begin{array}{l}0.069 \\
0.010 \\
0.016 \\
0.005 \\
0.005\end{array}$ & $\begin{array}{c}25.28 \\
9.18 \\
14.61 \\
2.29 \\
0.22\end{array}$ & $\begin{array}{l}79.3 \\
95.5 \\
99.3 \\
93.8 \\
95.8\end{array}$ & $\begin{array}{c}20.7 \\
4.5 \\
0.7 \\
6.2 \\
4.2\end{array}$ \\
\hline $\begin{array}{r}\text { 11'500 Jahre alter } \\
\text { Podzol**: } \\
0-10 \\
10-16 \\
16-24 \\
24-55 \\
55-105 \\
>105\end{array}$ & $\begin{array}{c}\mathrm{O} \\
\mathrm{E} \\
\mathrm{Bhs} \\
\mathrm{Bs} \\
\mathrm{Bw} \\
\mathrm{C}\end{array}$ & $\begin{array}{l}3-10 \\
10-16 \\
16-22 \\
24-34 \\
55-65 \\
105\end{array}$ & $\begin{array}{l}21.8 \\
75.6 \\
54.5 \\
61.3 \\
67.8 \\
68.7\end{array}$ & $\begin{array}{l}0.77 \\
0.15 \\
0.46 \\
0.34 \\
0.46 \\
0.56\end{array}$ & $\begin{array}{l}0.24 \\
0.57 \\
1.05 \\
1.51 \\
1.63 \\
1.69\end{array}$ & $\begin{array}{l}0.31 \\
1.00 \\
1.05 \\
1.82 \\
2.35 \\
2.47\end{array}$ & $\begin{array}{l}0.71 \\
3.13 \\
2.48 \\
2.91 \\
2.93 \\
3.38\end{array}$ & $\begin{array}{l}0.01 \\
0.01 \\
0.03 \\
0.05 \\
0.06 \\
0.06\end{array}$ & $\begin{array}{l}1.25 \\
1.33 \\
7.46 \\
5.54 \\
4.66 \\
4.25\end{array}$ & $\begin{array}{l}3.72 \\
10.47 \\
11.88 \\
14.61 \\
13.18 \\
15.01\end{array}$ & $\begin{array}{l}0.51 \\
1.23 \\
1.09 \\
0.89 \\
0.89 \\
0.83\end{array}$ & $\begin{array}{l}0.027 \\
0.020 \\
0.011 \\
0.011 \\
0.012 \\
0.005\end{array}$ & $\begin{array}{l}67.84 \\
3.66 \\
15.43 \\
5.66 \\
1.25 \\
0.22\end{array}$ & $\begin{array}{l}97.1 \\
97.1 \\
95.4 \\
94.6 \\
95.2 \\
97.1\end{array}$ & $\begin{array}{l}2.9 \\
2.9 \\
4.6 \\
5.4 \\
4.8 \\
2.9\end{array}$ \\
\hline
\end{tabular}

*beinhaltet zumeist Adsorptions- oder Kristallwasser

** Org. Substanz $=$ org. C $\cdot 1.72$

*** Nomenklatur gemäss FAO-UNESCO

Tab. 2: Totalgehalte der gesamten Bodenmatrix (Feinerde (Durchmesser $<2 \mathrm{~mm})+$ Skelett $(>2 \mathrm{~mm})$ ) in OxidForm

Total analysis of the bulk material including fine earth (diameter $<2 \mathrm{~mm}$ ) and soil skeleton $(>2 \mathrm{~mm})$ of the investigated soils represented as oxides

Composition chimique du sol (représentée par des oxydes) comprenant la terre fine (diamètre $<2 \mathrm{~mm}$ ) et grossière $(>2 \mathrm{~mm})$

\begin{tabular}{|c|c|c|c|c|c|c|c|c|c|c|}
\hline \multirow{2}{*}{$\begin{array}{l}\text { Bodenprofil } \\
\text { Horizontbereich }(\mathrm{cm})\end{array}$} & \multirow[b]{2}{*}{ Horizont } & \multirow[b]{2}{*}{$\varepsilon_{\mathrm{i}, \mathrm{w}}$} & \multicolumn{8}{|c|}{$\tau$} \\
\hline & & & $\mathrm{Si}$ & $\mathrm{Ca}$ & $\mathrm{Mg}$ & $\mathrm{K}$ & $\mathrm{Na}$ & $\mathrm{Al}$ & $\mathrm{Fe}$ & $\mathrm{Mn}$ \\
\hline \multicolumn{11}{|c|}{ 3'500 Jahre alter Haplic Podzol* } \\
\hline $0-8$ & $\mathrm{O}$ & 1.57 & -0.55 & -0.43 & -0.75 & -0.53 & -0.68 & -0.55 & -0.76 & -0.90 \\
\hline $8-15$ & E & 0.78 & -0.33 & -0.62 & -0.56 & -0.33 & -0.54 & -0.35 & -0.42 & -0.70 \\
\hline $15-22$ & Bhs & 0.18 & -0.22 & -0.44 & -0.44 & -0.29 & -0.52 & -0.34 & -0.15 & -0.56 \\
\hline $22-60$ & Bws & 0.12 & -0.01 & -0.16 & -0.03 & -0.05 & -0.14 & 0.07 & 0.09 & 0.25 \\
\hline$>60$ & $\mathrm{C}$ & 0.00 & 0.00 & 0.00 & 0.00 & 0.00 & 0.00 & 0.00 & 0.00 & 0.00 \\
\hline \multicolumn{11}{|c|}{11 '500 Jahre alter Haplic Podzol* } \\
\hline $0-10$ & $\mathrm{O}$ & 6.12 & -0.48 & 1.27 & -0.77 & -0.66 & -0.80 & -0.60 & -0.52 & -0.82 \\
\hline $10-16$ & E & 0.06 & -0.25 & -0.81 & -0.77 & -0.37 & -0.73 & -0.53 & -0.79 & -0.90 \\
\hline $16-24$ & Bhs & 0.28 & -0.39 & -0.36 & -0.53 & -0.44 & -0.67 & -0.39 & 0.35 & -0.65 \\
\hline $24-55$ & Bs & 0.69 & -0.17 & -0.43 & -0.17 & -0.20 & -0.31 & -0.09 & 0.22 & -0.26 \\
\hline $55-105$ & $\mathrm{Bw}$ & 0.04 & -0.08 & -0.22 & -0.10 & -0.19 & -0.11 & -0.18 & 0.03 & -0.04 \\
\hline$>105$ & C & 0.00 & 0.00 & 0.00 & 0.00 & 0.00 & 0.00 & 0.00 & 0.00 & 0.00 \\
\hline
\end{tabular}

*Die Nomenklatur der Bodenhorizonte richtet sich nach der FAO-UNESCO

Tab. 3: Strain Koeffizienten $\left(\varepsilon_{\mathrm{i}, \mathrm{w}}\right)$ und relative Massenverluste $(\tau)$ für jedes untersuchte Element in Abhängigkeit der Bodentiefe

Strain coefficients $\left(\varepsilon_{i, w}\right)$ and relative losses $(\tau)$ of every investigated element with respect to the soil depth Coefficients de compaction (valeurs négatives) et de dilatation (valeurs positives) $\varepsilon_{i, w}$ et pertes relatives de masse $(\tau)$ pour chaque élément en relation avec la profondeur du sol 


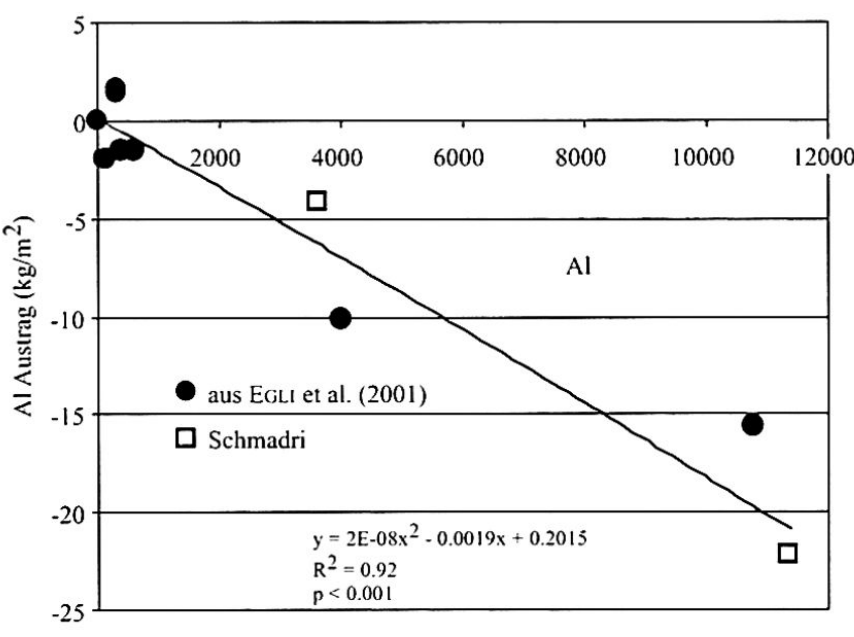

Bodenalter (Jahre)

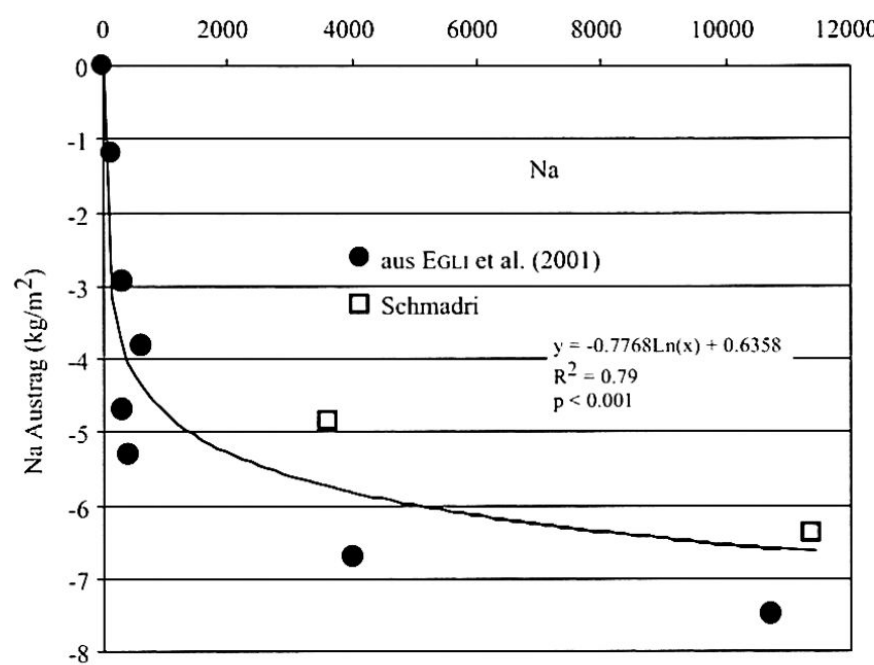

Bodenalter (Jahre)

Abb. 5: Verluste an Al und Na im Verlaufe der Zeit. Die Daten wurden mit Resultaten aus EgLI et al. (2001) ergänzt und mit Trendlinien versehen.

Weathering losses of $\mathrm{Al}$ and $\mathrm{Na}$ as a function of time. The data has been completed with results from EGLI et al. (2001) and with corresponding regression curves.

Pertes en Al et en Na en relation avec l'âge du sol. Les données ont été complétées par les résultats d'EGLI et al. (2001) et sont pourvues de régressions.

$\mathrm{Mg}$ und $\mathrm{K}$ sind beim älteren Podsol meist wesentlich bedeutender. Ähnliche Verluste innerhalb einer gleichen Zeitspanne der Bodenentwicklung zeigen auch Untersuchungen von JERSAK et al. (1995) oder OLSSON \& Melkerud (2000). Charakteristisch für Podsole sind zudem die Al Austräge, wobei wiederum der ältere Podsol bis in grössere Tiefen deutlich negativere Werte hat. Werden die Verlustraten gegenüber der Zeit aufgetragen und mit Daten aus einem ähnlich situierten Gebiet (Gletsch: ähnliche Geologie, ähnliches Klima) verglichen, so erkennt man, dass der Verlust von $\mathrm{Al}$ - im Gegensatz zu Na - eher einen linearen Charakter aufweist und der jüngere Podsol im Schmadrigebiet auch tatsächlich beträchtlich geringere Al-Verluste verzeichnet als der ältere (Abb. 5). Natrium wird vor allem zu Beginn der Bodenbildung (<3'000 Jahre) sehr intensiv ausgetragen. Beim Fe sind die Prozesse der Eluviation und Illuviation beim älteren Podsol klar sichtbar (Tab. 3), währenddem beim jüngeren dies weniger deutlich ausfällt. Daraus lässt sich schliessen, dass $\tau_{j, w}$ der untersuchten Elemente mit zunehmendem Ailter des Bodens profilumfassend negativer wird und sich zur Unterscheidung von Böden unterschiedlichen Alters gut eignen kann.

\subsection{Mineralverwitterung und -neubildung}

Normative Berechnungen der Gesamtmineralogie zeigen den granitischen Charakter des Ausgangsmaterials. Die Hauptminerale sind Albit, Orthoklas, Anor- thit, Muskovit, Biotit (+Illit) und Quarz. Im Verlauf des Verwitterungsprozesses werden vor allem Albit bzw. Plagioklase, K-Feldspäte und Biotite bzw. Illite aufgelöst oder umgewandelt. Als Sekundärprodukte erscheinen neue Schichtsilikate (wie bspw. Vermiculite), Al-Hydroxide, Allophane oder Imogolite sowie etwas Goethit (Abb. 6). Die Röntgendiffraktogramme der Tonfraktion weisen auf ganz interessante Eigenschaften hin. Das Ausgangsmaterial (C-Horizont) wird durch enge und gut ausgebildete Peaks charakterisiert. Die Peaks bei $1.43,1.0$ und $0.71 \mathrm{~nm}$ lassen auf Chlorit, Biotite (+Illit) und Kaolinit schliessen (MOORE \& REYNOLDS 1997). Mittels IR Analysen wird Kaolinit jedoch nicht nachgewiesen. Somit entfällt der Peak bei 0.71 nm auf die $d(002)$ Reflektion eines Fe-reichen Chlorits. Der kleinere Peak bei $0.64 \mathrm{~nm}$ beschreibt Plagioklas. Die engen Peaks deuten auf eine gut ausgebildete Kristallisation hin. Eluvialhorizonte von Podsolen sind vom mineralogischen Standpunkt am intensivsten verwittert. Im E-Horizont des jüngeren Podsols ( 3500 Jahre) erkennt man eine bereits völlig andere mineralogische Zusammensetzung. Im Diffraktogramm der mit Ethylen Glykol behandelten Probe lassen sich Peaks bei den $d$-Werten $2.65,1.70,1.34,1.22,1.0$, 0.93, 0.71 und $0.67 \mathrm{~nm}$ bestimmen (Abb. 7). Biotit und Illit werden durch den Schichtabstand $d=1.0$ $\mathrm{nm}$ beschrieben. Die Werte 2.65, 1.34 und $0.93 \mathrm{~nm}$ entsprechen den $d(001), d(002)$, und $d(003)$ Reflektionen einer regelmässigen Wechsellagerung von Mica/ 


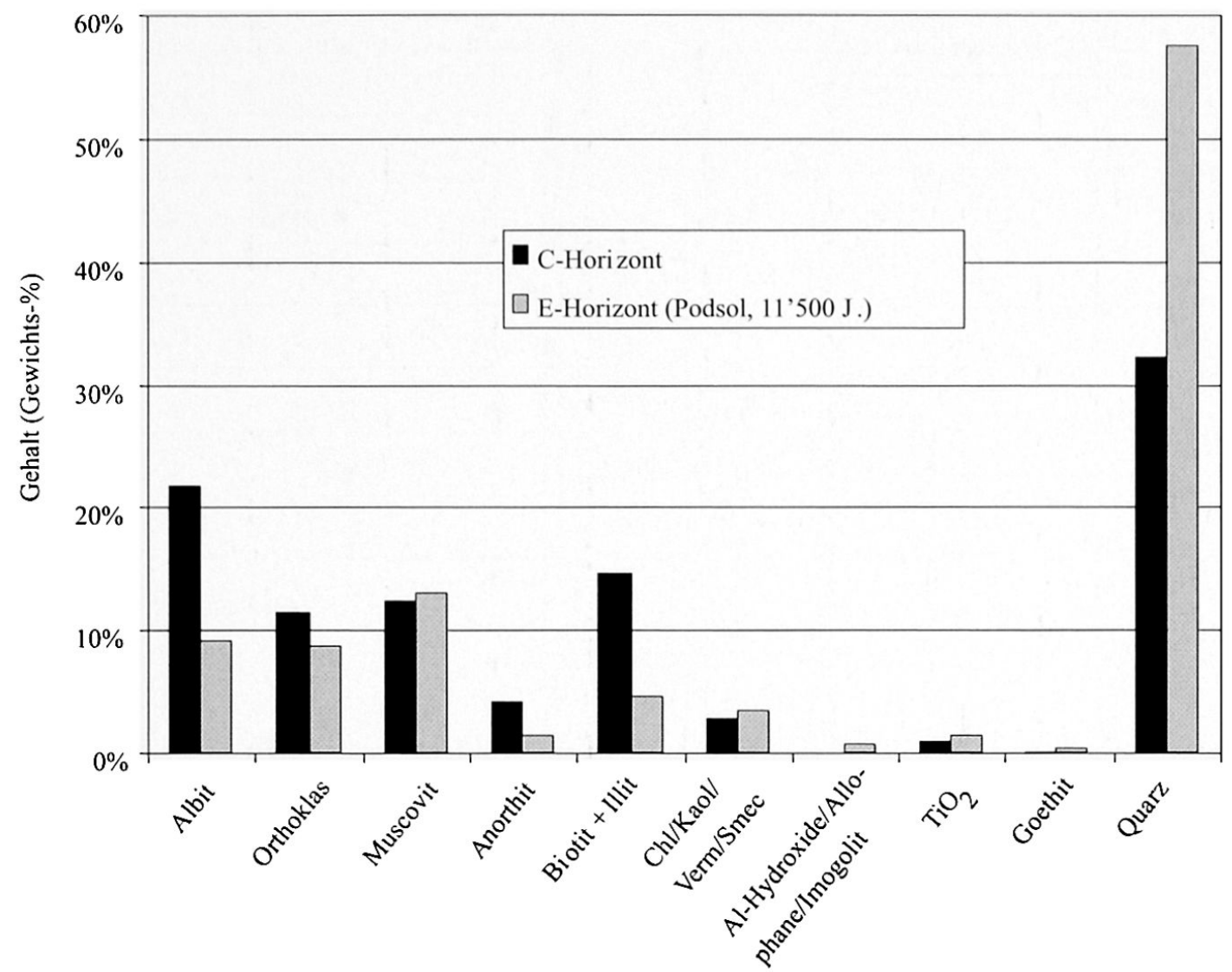

Abb. 6: Approximativer Gehalt an Primär- und Sekundärmineralien in den untersuchten Böden Estimated mean mineral composition (primary and secondary minerals) in the investigated soils Contenu approximatif en minéraux (minéraux primaires et secondaires) dans les sols étudiés

\begin{tabular}{|c|c|c|c|c|c|c|c|c|c|c|}
\hline $\begin{array}{l}\text { Bodentiefe }(\mathrm{cm}) \\
\text { untersuchte Horizonte }\end{array}$ & & Biotit/Illit & Chlorit & Vermiculit & $\begin{array}{l}\text { Hydrobiotit } \\
\text { V/I* }\end{array}$ & Smectit & $\begin{array}{l}\text { Smectit/ } \\
\text { Mica++ }\end{array}$ & HIS + & Kaolinit & Plagioklas \\
\hline \multicolumn{11}{|c|}{ 3'500 Jahre alter Haplic Podzol** } \\
\hline $8-15$ & $\mathrm{E}$ & $\mathrm{x}$ & & $\mathrm{x}$ & & $\mathrm{x}$ & $\mathrm{x}$ & $(\mathrm{x})^{\#}$ & (x) & $\mathrm{x}$ \\
\hline $15-22$ & Bhs & $\mathrm{x}$ & $\mathrm{x}$ & $\mathrm{x}$ & $\mathrm{x}$ & & & & (x) & $\mathrm{x}$ \\
\hline$>60$ & $\mathrm{C}$ & $\mathrm{x}$ & $\mathrm{x}$ & & & & & & & $\mathrm{x}$ \\
\hline \multicolumn{11}{|c|}{11 '500 Jahre alter Haplic Podzol } \\
\hline $10-16$ & E & $\mathrm{x}$ & & $\mathrm{x}$ & & $\mathrm{x}$ & $\mathrm{x}$ & & (x) & $\mathrm{x}$ \\
\hline $16-24$ & Bhs & $\mathrm{x}$ & $\mathrm{x}$ & $\mathrm{x}$ & $\mathrm{x}$ & & & (x) & (x) & $\mathrm{x}$ \\
\hline $55-105$ & $\mathrm{Bw}$ & $\mathrm{x}$ & $\mathrm{x}$ & & & & & & & $\mathrm{x}$ \\
\hline$>105$ & C & $\mathrm{x}$ & $\mathrm{x}$ & & & & & & & $\mathrm{x}$ \\
\hline
\end{tabular}

$* \mathrm{~V} / \mathrm{I}=$ Vermiculit/ Illit Wechsellagerung

+ HIS $=$ Hydroxy interlayered smectites

\# () in geringen Mengen vorhanden, vermutet
** Nomenklatur gemäss FAO-UNESCO

++ Mica: Illit, Biotit oder Muskovit. Hier dioktaedrischer Mica (Muskovit)

Tab. 4: Mineralogische Zusammensetzung der Tonfraktion: Überblick 

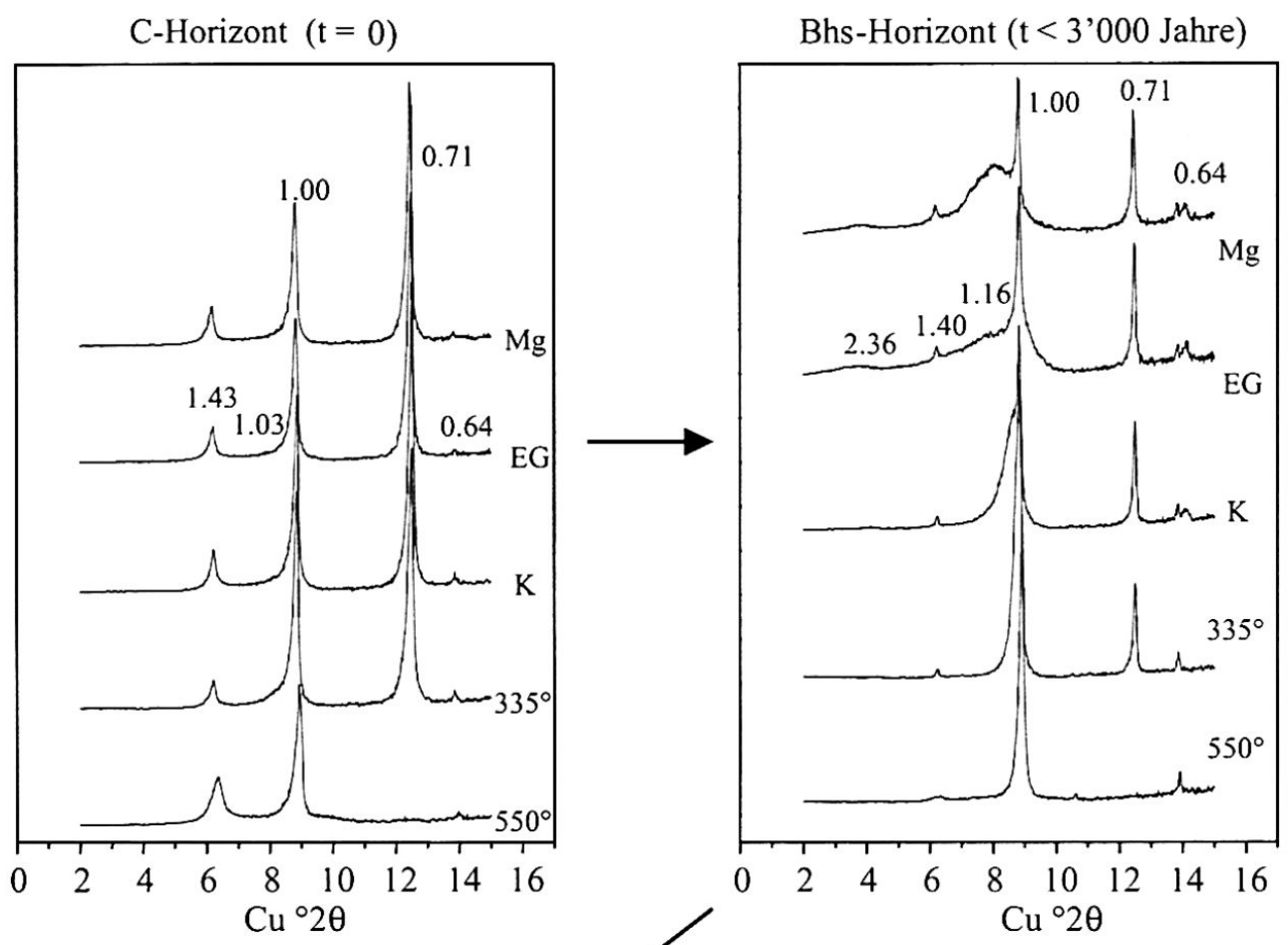

E-Horizont (junger Podsol, $\mathrm{t}=\mathrm{ca}$. 3'500 Jahre)

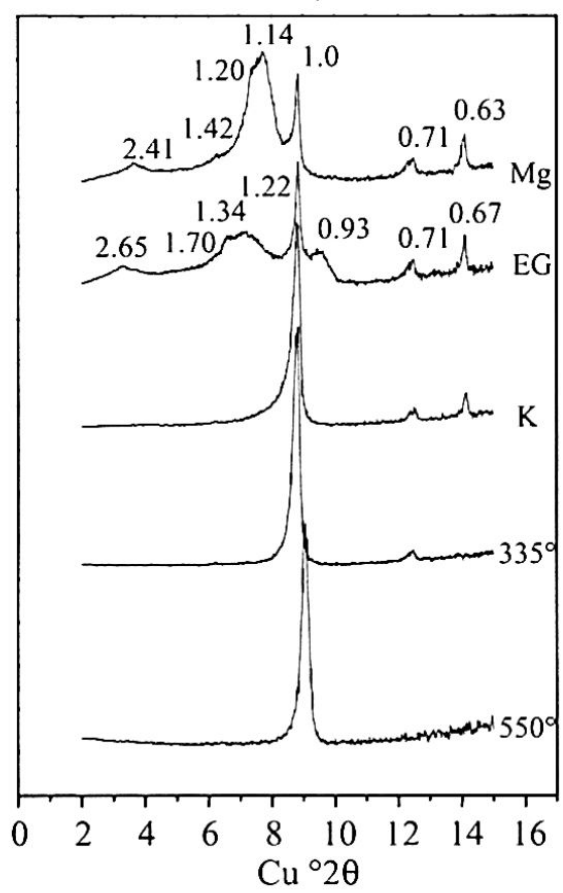

E-Horizont (alter Podsol, $\mathrm{t}=\mathrm{ca} .11$ '500 Jahre)

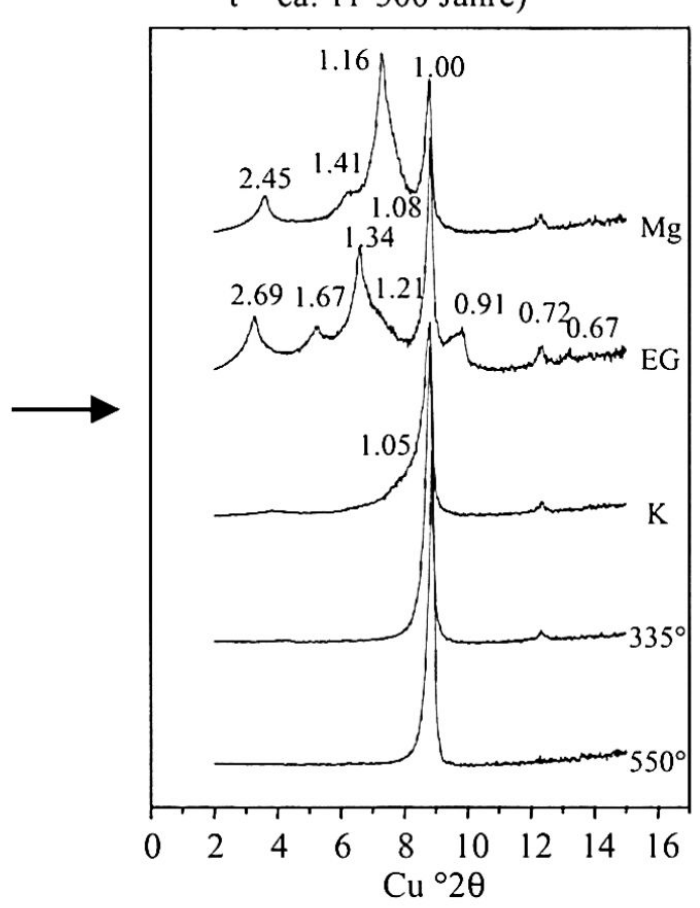

Abb. 7: Zeitliche Entwicklung der Mineralien in der Tonfraktion. Die Schichtabstände sind in nm angegeben. $\mathrm{Mg}=$ Sättigung mit $\mathrm{Mg}, \mathrm{EG}=$ Bedampfung mit Ethylen Glykol, $\mathrm{K}=$ Sättigung mit $\mathrm{K}, 335^{\circ}=$ Erhitzen bei $335^{\circ} \mathrm{C}$, $550^{\circ}=$ Erhitzen bei $550^{\circ} \mathrm{C}$

Temporal evolution of soil clay minerals. The d-spacings are given in $\mathrm{nm} . M g=M g$ saturation, $E G=$ ethylene glycol solvation, $K=K$ saturation, $335^{\circ}=$ heating at $335^{\circ} \mathrm{C}, 550^{\circ}=$ heating at $550^{\circ} \mathrm{C}$

Evolution temporelle des minéraux de la fraction argileuse. L'écart entre les couches est donné en $\mathrm{nm}$. $\mathrm{Mg}=$ saturation en $\mathrm{Mg}, E G=$ solution avec éthylène glycol, $335^{\circ}=$ chauffage à $335^{\circ} \mathrm{C}, 550^{\circ}=$ chauffage à $550^{\circ} \mathrm{C}$ 

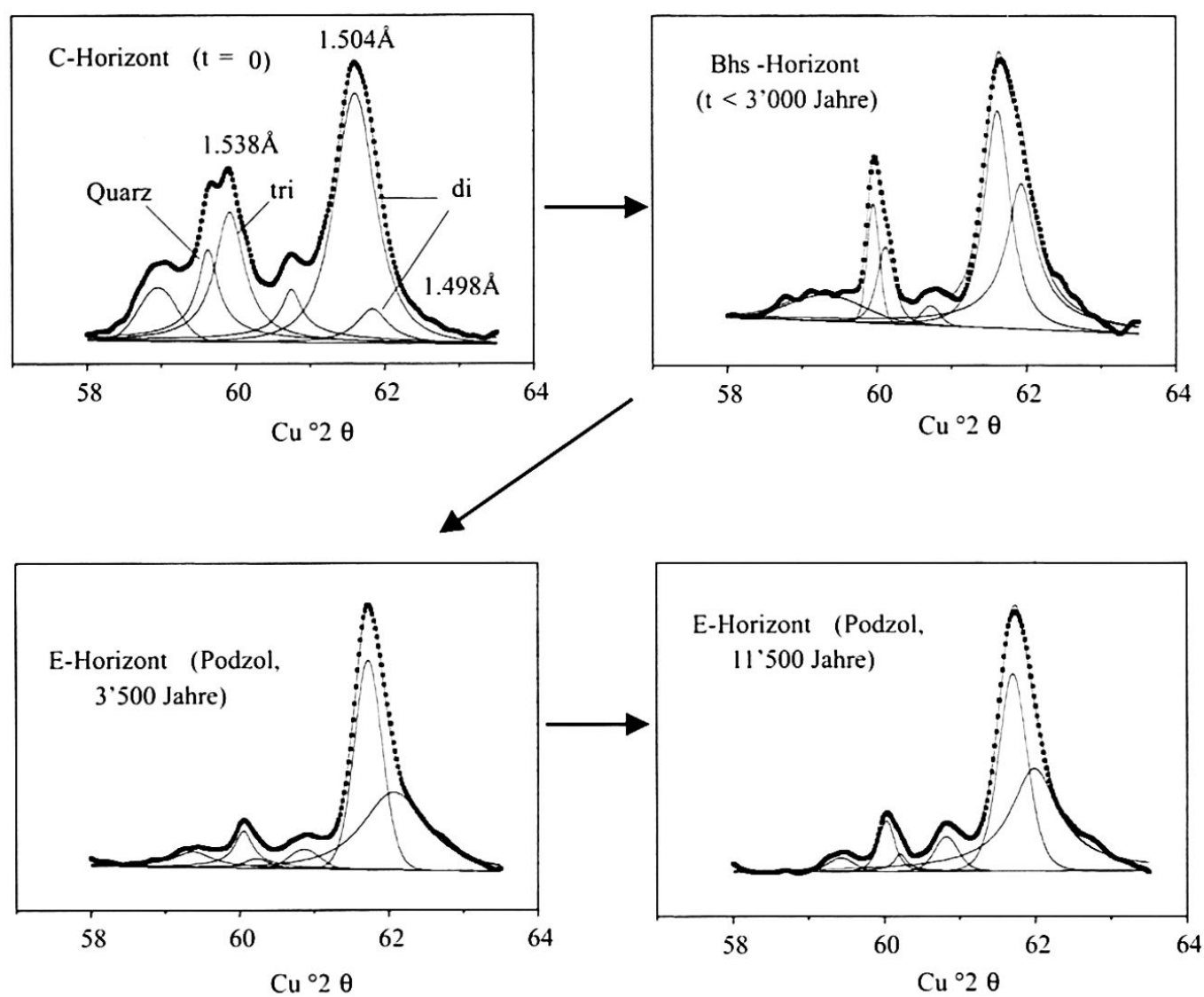

Abb. 8: Analyse der d(060) Region (Tonfraktion) vom Ausgangsmaterial (C-Horizont) bis hin zum E-Horizont des 11'500 Jahre alten Podsols; di = Peaks für dioktaedrische Mineralien, tri = Peak für trioktaedrische Mineralien

$X$-ray patterns in the $\mathrm{d}(060)$ region of the clay fraction with respect to its temporal evolution: from the $C$ horizon up to the E horizon of the 11'500 years old podzol; di = dioctahedral minerals, tri = trioctahedral minerals

Diffractogramme de rayons $X$ dans la région $\mathrm{d}(060)$ des minéraux de la fraction argileuse en relation avec l'évolution temporelle (de l'horizon $C$ à l'horizon E du podzol de 11'500 ans); di = minéraux dioctaédriques, tri = minéraux trioctaédriques

\begin{tabular}{|c|c|c|c|}
\hline $\begin{array}{c}\text { Ausgangsmaterial } \\
\text { C-Horizont } \\
\mathrm{t}=0\end{array}$ & $\begin{array}{l}\text { Bhs-Horizont } \\
\mathrm{t}<3 \text { '000 Jahre }\end{array}$ & $\begin{aligned} & \text { E-Horizont } \\
\mathrm{t}= & \mathrm{ca} . \text { 3'500 Jahre }\end{aligned}$ & $\begin{array}{c}\text { E-Horizont } \\
\mathrm{t}=\mathrm{ca} .11 \text { '500 Jahre }\end{array}$ \\
\hline $\begin{array}{l}\text { Biotit, } \\
\text { Illit }\end{array}$ & $\begin{array}{l}\text { Illit/Vermiculit } \\
\text { (Hydrobiotit) }\end{array}$ & Mica/Smectit & Mica/Smectit \\
\hline Chlorit & (HIS) & (HIS) & Smectit \\
\hline
\end{tabular}

Abb. 9: Beobachtete Mineraltransformationen und deren zeitliche Einordnung Observed mineral transformations during the soil evolution Transformations des minéraux observées durant la genèse du sol $H I S=$ Hydroxy interlayered smectites 
Smectit. Diese Wechsellagerung besteht zu rund $65 \%$ aus Mica (Illit, Biotit oder Muskovit) und $35 \%$ aus Smectit. Smectit selber wird durch den Peak bei 1.70 $\mathrm{nm}$ angezeigt. Das Verschwinden des Peaks bei 1.4 $\mathrm{nm}$ nach dem Erhitzen deutet auf Vermiculit-ähnliche Mineralien hin bzw. auf die Abwesenheit von Chlorit. Ebenso verschwindet der Peak bei 0.71 nach dem Heizen vollständig und kann somit Kaolinit zugeordnet werden. Die Ausprägung der Peaks ist, abgesehen vom Biotit/Illit-Peak, breiter und oft nicht mehr so deutlich wie beim Ausgangsmaterial. Die Kristallisation der einzelnen Mineralien ist somit wesentlich schlechter. Ein weiterer Schritt in der Bodenentwicklung widerspiegelt sich im E-Horizont des älteren Podsols. Dieselben Mineralien wie beim jüngeren Podsol sind erkennbar. Die Peaks sind jedoch wesentlich deutlicher und enger, was auf eine bessere Kristallisation schliessen lässt. Die Abfolge der Peaks bei 2.69, 1.34 und $0.91 \mathrm{~nm}$ deutet auf eine regelmässige Wechsellagerung mit ähnlichen Anteilen von Mica und Smectiten (Moore \& ReYnolds 1997) hin. Die Behandlung der Proben mit Na-Citrat verursachte nur beim Diffraktogramm des Bhs-Horizontes des älteren Podsols substantielle Änderungen. Geringfügige Änderungen konnten ebenfalls für den E-Horizont des jüngeren Podsols ausgemacht werden. In diesen Horizonten lassen sich Zwischenschichten bestehend aus Hydroxiden von $\mathrm{Al}, \mathrm{Fe}$ und $\mathrm{Mg}$ in Tonmineralien nachweisen. Im Bhs-Horizont des älteren Podsols finden sich hydroxy-interlayered-smectites (HIS), währenddem im E-Horizont des jüngeren Podsols diese nur noch in geringen Mengen vorhanden waren (Tab. 4). Die Bhs-Horizonte beider Podsole haben ansonsten sehr ähnliche Minerale. Die Schichtabstände von $d(001)$ bei ca. $2.3 \mathrm{~nm}$ und $1.15 \mathrm{~nm}$ entsprechen einer regelmässigen Wechsellagerung zwischen Vermiculit und Illit, welche für Hydrobiotit typisch ist (SAwhNey 1967, Moore \& REYNOLDS 1997). Zusätzlich können Vermiculit, Biotit (+Illit) und Kaolinit beobachtet werden. Smectit sowie eine mögliche Wechsellagerung mit Mica ist nicht mehr nachweisbar. Ganz im Gegensatz zum E-Horizont beider Podsole findet sich wiederum Chlorit (vgl. Tab. 4).

Die Analyse der $d(060)$ Region der Tonfraktion aller Horizonte zeigt (Abb. 8), dass der aus trioktaedrischen Mineralien resultierende Peak (bei $1.544 \AA$ Å) im C-Horizont im Vergleich zu den Verwitterungshorizonten wesentlich grösser ist. Je weiter die Verwitterung fortschreitet, desto unbedeutender werden die trioktaedrischen Mineralien bzw. desto mehr dioktaedrische Mineralien (zwei Peaks um $1.500 \AA$ ) lassen sich nachweisen. Diese Evolution weist auf eine kontinuierliche Transformation von trioktaedrischen zu dioktaedrischen Strukturen hin, mit einer wachsenden Bedeutung von aufweitbaren 2:1 Schichtsilikaten. Aufgrund der starken Abnahme des Peaks bei $1.534 \AA$ und einer entsprechenden Zunahme bei $1.491 \AA$ sowie dem Verharren des $1.504 \AA$ Peaks kann beim Mineral Mica/Smectit, das im Verlaufe der Bodenbildung entsteht, auf eine Wechsellagerung von dioktaedrischem Mica (Muskovit) mit Smectit geschlossen werden. Die E-Horizonte beider Podsole haben ein sehr ähnliches Spektrum, woraus geschlossen werden kann, dass der Transformationsprozess von trioktaedrischen zu dioktaedrischen Strukturen nach rund 3'500 Jahren zu einem Grossteil bereits abgeschlossen ist. In beiden Podsolen verschwinden mit zunehmender Tiefe allmählich die aufweitbaren Minerale. Als Gegensatz dazu lässt sich eine kontinuierliche Zunahme von Chlorit beobachten. Daraus kann der Schluss gezogen werden, dass sich Smectite aus der Verwitterung von Chloriten bilden. Die Wechsellagerung von Smectit mit Mica und von Mica (bzw. Illit) mit Vermiculit (Hydrobiotit) deutet darauf hin, dass Smectit auch aus Biotit bzw. Illit gebildet wird und zwar über die Zwischenstufe Vermiculit. Je älter der Boden und je weiter die Verwitterungsprozesse abgelaufen sind, desto besser ist die Kristallisation und Ausprägung der neugeformten Sekundärprodukte. Die zeitliche Abfolge dieser Prozesse lässt sich zusammenfassend aus Abb. 9 entnehmen.

\section{Schlussfolgerungen}

Obwohl an den untersuchten Standorten der gleiche Bodentyp vorgefunden werden kann (Podsol), lässt sich aufgrund des chemischen und mineralogischen Verwitterungsgrades eine eindeutige Unterscheidung vornehmen. Der jüngere Podsol, dessen Alter mittels gletschergeschichtlichen Methoden auf ca. 3'500 Jahre beziffert werden kann, weist vom chemischen wie mineralogischen Standpunkt her einen niedrigeren Verwitterungs- bzw. Entwicklungsgrad auf. Dies lässt sich am Beispiel der $\mathrm{Al}_{\mathrm{d}} / \mathrm{Fe}_{\mathrm{d}}$-Verhältnisse, der gespeicherten $\mathrm{N}$-Menge, der Al- und $\mathrm{Na}$-Verwitterung sowie auch bei der Tonmineralogie beobachten. Die Ergebnisse aus den bodenchemischen Untersuchungen lassen sich sehr gut in Chronosequenzen aus Gebieten mit ähnlicher Geologie, Topographie und Klima einordnen. Die $\mathrm{Al}_{\mathrm{d}} /$ $\mathrm{Fe}_{\mathrm{d}}$-Verhältnisse, die gespeicherte $\mathrm{N}$-Menge und die Al- und Na-Verwitterung scheinen somit auch im überregionalen Rahmen brauchbare Kriterien zu sein, um den Verwitterungsgrad von Böden relativ exakt einordnen zu können. Die Berechnungen mit Verwitterungsindizes widerspiegeln zwar die Tendenzen der Verwitterungsprozesse. Sie sind aber für eine präzisere zeitliche Zuordnung von Böden - zumindest für die in Betracht gezogenen Untersuchungsgebiete - weniger geeignet. Die Verwitterungssequenz bei den Tonmineralien zeigt deutlich auf, dass die entscheidenden Veränderungen innerhalb der ersten rund 3'000 Jahre ablaufen. Diese Ergebnisse sind im Einklang mit Resultaten von RigHI et al. (1999). Die Endprodukte 
der Verwitterung sind Smectite und Wechsellagerungsminerale mit Smectit und Mica, die sich im wesentlichen aus Chlorit bzw. Illit/Biotit gebildet haben. Die Zusammensetzung der Tonfraktion widerspiegelt in diesem Fall die Bodenentwicklung sehr deutlich.

\section{Dank}

Die Arbeiten wurden durch die Kommission zur Förderung des akademischen Nachwuchses (Universität Zürich), den Consiglio Nationale delle Ricerche (CNR, Italien) und den Schweizerischen Nationalfonds finanziell unterstützt. Zwei ungenannten Gutachtern danken wir für die konstruktiven Hinweise.

\section{Literatur}

BorgGaARD, O.K. (1988): Phase indication by selective dissolution techniques. - In: STUCKI, J. W., GoodmaN, B.A. \& U. Schwertmann (eds): Iron in Soils and Clay Minerals. - D. Reidel Publishing Company, Dordrecht: 83-89.

Bäumler, R., Zech, W., Heuberger, H. \& K. WeberDIEFENBACH (1991): Investigations on the intensity of weathering of soils developed from glacial and fluvioglacial deposits and their relationship with the history of the landscape in the Mt. Everest region. - In: Geoderma 48: 223-243.

Bäumler, R., Kemp-Oberhettinger, M., Zech, W., Heuberger, H., Siebert, A., Madhikarmi, D.P. \& K.P. Poudel (1996): Soil weathering on glacial and fluvioglacial deposits in the Langtang Valley (Central Nepal) and its relation to the glacial history. - In: Zeitschrift für Geomorphologie, Supplementband 103: 373-387.

Burga, C. \& R. Perret (1998): Vegetation und Klima der Schweiz seit dem jüngeren Eiszeitalter. - Thun: Ott Verlag.

Chadwick, O.A., Brimhall, G.H. \& D.M. Hendricks (1990): From a black to a gray box - a mass balance interpretation of pedogenesis. - In: Geomorphology 3: 369-390.

DAhlgren, R.A. \& F.C. Ugolini (1991): Distribution and characterization of short-range-order minerals in Spodosols from the Washington Cascades. - In: Geoderma 48: 391-413.

Egli, M. \& P. Fitze (2000): Formulation of pedologic mass balance based on immobile elements: a revision. - In: Soil Science 165: 437-443.

Egli, M., Fitze, P. \& A. Mirabella (2001): Weathering and evolution of soils formed on granitic, glacial deposits: results from chronosequences of Swiss alpine environments. - In: Catena 45: 19-47.

EgGENBERGER, U. (1995): Mineral weathering in soils: experiments, field studies, and modeling. $-=$ Ph.D. Thesis, University of Berne, Switzerland.

FITZE, P.F. (1982): Zur Relativdatierung von Moränen aus der Sicht der Bodenentwicklung in den kristallinen Zentralalpen. - In: Catena 9:265-306.

Fitze, P., KäGI, B. \& M. EgLI (2000): Laboranleitung zur Untersuchung von Boden und Wasser. $-=$ Geographisches Institut der Universität Zürich, Zürich, Switzerland.

Hardy, M., Jamagne, M., Elsass, F., Robert, M. \& D. Chesneau (1999): Mineralogical development of the silt fractions of a podzoluvisol on loess in the Paris Basin (France). - In: European Journal of Soil Science 50: 443-456.

HarnoIs, L. (1988): The CIW index: a chemical index of weathering. - In: Sedimentary Geology 55: 319-322. JenNy, H. (1941): Factors of soil formation. - New York: Mc Graw-Hill Book Company.

Jenny, H. (1980). The soil resource. - New York: Springer Verlag.

Jersak, J., Amundson, R. \& G. Brimhall (1995): A mass balance analysis of podzolization: examples from the northeastern United States. - In: Geoderma 66: $15-42$.

Keller, O. (1994): Entstehung und Entwicklung des Bodensees - Ein geologischer Lebenslauf. - In: H. Maurer (Hrsg.): Umweltwandel am Bodensee. - UVK Fachverlag für Wissenschaft und Studium GmbH, St. Gallen: 33-92.

KRONBERG, G.I. \& H.W. NesbitT (1981): Quantification of weathering of soil chemistry and soil fertility. - In: Journal of Soil Science 32: 453-459.

Lichter, J. (1998): Rates of weathering and chemical depletion in soils across a chronosequence of Lake Michigan sand dunes. - In: Geoderma 85: 255-282.

Liski, J., IlvesNiemi, H., MÄKELÄ, A. \& M. STARR (1998): Model analysis of the effect of soil age, fire and harvesting on the carbon storage of boreal forest soils. In: European Journal of Soil Science 49: 407-416.

MaISCH, M. (2000): The longterm signal of climate change in the Swiss Alps: glacier retreat since the end of the little ice age and future ice decay scenarios. - In: Geografia Fisica e Dinamica Quaternaria 23: 139-155. Mirabella, A., Constantini, E.A.C. \& S. Carnicelli, (1992): Genesis of a polycyclic Terra Rossa (chromic cambisol on rhodic nitisol) at the Poggio del Commune in Central Italy. - In: Zeitschrift für Pflanzenernährung und Bodenkunde 155: 407-413.

Moore, D.M. \& R.C. ReYNolds (1997): X-ray diffraction and the identification and analysis of clay minerals. $-2^{\text {nd }}$ edition, New York: Oxford University Press. Nesbitt, H.W. \& G.M. Young (1989): Formation and diagenesis of weathering profiles. - In: Journal of Geology 97: 129-147.

OlsSON, M.T. \& P.-A. MelKerud (2000): Weathering in three podzolized pedons on glacial deposits in northern Sweden and central Finland. - In: Geoderma 94: 149-161.

Parfitt, R.L. \& T. Hemni (1982): Comparison of an oxalate-extraction method and an infrared spectrosco- 
pic method for determining allophane in soil clays. In: Soil Science and Plant Nutrition 28: 183-190.

REICHE, P. (1943): Graphic representation of chemical weathering. - In: Journal of Sedimentary Petrology 13: 58-68.

Righi, D., Huber, K. \& C. Keller (1999): Clay formation and podzol development from postglacial moraines in Switzerland. - In: Clay Minerals 34: 319-332.

SAWHNEY, B.L. (1967): Interstratification in vermiculite. - In: Clays and Clay Minerals 15: 75-84.

SHOJI, S. \& Y. FuJIWARA (1984): Active aluminium and iron in the humus horizons of andosols from northeastern Japan: their forms, properties, and significance in clay weathering. - In: Soil Science 137: 216-226.

TAMURA, T. (1958): Identification of clay minerals from acid soils. - In: Journal of Soil Science 9: 141-147.

Theurillat, J.P., Felber, F., Geissler, P., Gobat, J.M., Fierz, M., Fischlin, A., KüPfer, P., SChlüssel, A., VelLuti, C., ZhaO, G.-F. \& J. Williams (1998): Sensitivity of plant and soil ecosystems of the alps to climate change. - In: Cebon, P. , Dahinden, U., Davies, H.C., ImBODEN, D. \& C. JAEger (eds): Views from the Alps. MIT Press, Massachusetts: 225-308.

Vogel, D.E. (1975): Precambrian weathering in acid metavolcanic rocks from the Superior Province, Villebon Township, southcentral Quebec. - In: Canadian Journal of Soil Science 12: 2080-2085.

WADA, K. (1977): Allophane and imogolite. - In: Dixon, J.B. \& S.B. WEED (eds): Minerals in soil environment. In: Soil Science Society of America, Madison, Wisconsin, USA: 603-638.

WIPF, A. (2001): Gletschergeschichtliche Untersuchungen im spät- und postglazialen Bereich des Hinteren Lauterbrunnentals (Berner Oberland, Schweiz). - In: Geographica Helvetica, diese Nummer.

Zusammenfassung: Bodenkundliche Untersuchungen im spät- und postglazialen Bereich des Hinteren Lauterbrunnentals Berner Oberland, Schweiz): Bodenchemischer und -mineralogischer Vergleich zweier Podsole auf unterschiedlich alten Moränen

Chronosequenzen vermitteln einen Einblick in die im Boden ablaufenden Prozesse und ermöglichen zudem Aussagen über die Landschaftsgenese. Die Verbindung des Faktors Zeit mit festgestellten Änderungen im Boden geben Hinweise über die Geschwindigkeit der entsprechenden Prozesse. Die im Schmadri untersuchten Bodenprofile (ca. 3'500 und 11'500 Jahre) zeigen eindeutige Unterschiede hinsichtlich ihres Entwicklungsgrades. Eine gute zeitliche Einordnung der Böden lässt sich aufgrund des dithionit-extrahierbaren $\mathrm{Fe}$ und $\mathrm{Al}$ bewerkstelligen. Auch die gespeicherte Menge an Stickstoff im Boden ist mit dem Bodenalter eng verknüpft. Ganz deutliche Unterschiede lassen sich über die Bestimmung der Verwitterungsraten berechnen. Die Verwitterungsraten von $\mathrm{Al}$ widerspiegeln im wesentlichen den Verlauf der Podsolierung, währenddem Na sehr stark die Verwitterung von Plagioklasen reflektiert. Eine gute zeitliche Abfolge ist auch bei den Tonmineralien erkennbar. Die Verwitterung läuft im wesentlichen über die Umwandlung von Chloriten und Glimmern hin zur Bildung von Hydrobiotit oder HIS und schliesslich zu Smectiten oder einer Wechsellagerung zwischen Smectit und Mica.

\section{Summary: Pedological Research in Late and Post- Glacial Areas of the Upper Lauterbrunnen Valley} (Bernese Oberland, Switzerland): Soil chemistry and soil mineralogy comparisons between podsols on two different historic moraines

Soil chronosequences give important insights into processes taking place in the soil and enable detailed descriptions of the development of landscapes. If the changes that have occurred in the soil can be connected to the state factor time then the velocity of these processes can be derived. The soil profiles (with a soil age of 3'500 and 11'500 years) in the investigation area Schmadri showed considerable differences with respect to their development stage. We found that dithionite-extractable $\mathrm{Fe}$ and $\mathrm{Al}$ are closely related to the duration of soil development. Furthermore, there is a close relationship between the mass of organic $\mathrm{N}$ in the whole profile and the soil age. The behaviour of $\mathrm{Al}$ illustrates classic patterns of podzolisation. $\mathrm{Na}$ is closely related to the weathering of plagioclase and shows substantial losses during soil pedogenesis. Additionally, a distinct temporal sequence can be observed for the clay minerals. The weathering reactions included to a great extent the transformation of chlorite and mica into hydrobiotite or hydroxy-interlayered smectites and finally into smectites or interlayered smectite/mica.

Résumé: Etudes pédologiques dans le domaine tardiet post-glaciaire du fond de la vallée de Lauterbrunnen (Hinteres Lauterbrunnetal, Oberland bernois, Suisse): comparaison de la chimie et de la minéralogie de deux podzols développés sur des moraines de différents âges

Les chronoséquences du sol donnent un bon aperçu des processus qui se déroulent dans le sol et témoignent de la genèse du paysage. En mettant en relation le facteur temps et les modifications observées dans le sol, on obtient des informations sur la rapidité des processus en question. Les profils pédologiques étudiés à Schmadri (environ 3'500 et 11'500 ans) présentent des différences nettes en ce qui concerne leur degré d'évolution. Grâce à l'extractibilité du fer et de l'aluminium par le dithionite, il est possible de classer ces sols dans le temps. La quantité d'azote stocké dans le sol est également étroitement liée à l'âge du sol. La détermination des taux de décomposition fait apparaître des 
différences bien marquées. Les taux de décomposition de l'aluminium reflètent le processus classique de la podzolisation, alors que le sodium est étroitement lié à la décomposition des plagioclases et montre des pertes substantielles durant la pédogenèse. De plus, on observe également une bonne séquence temporelle chez les minéraux de la fraction argileuse. La décomposition va da la transformation des chlorites et des micas à la formation d'hydrobiotite ou d'HIS (hydroxy-interlayered smectites) et, finalement, à la formation de smectites ou à une stratification de couches de smectites et de micas.

Dr. Markus Egli, Geographisches Institut, Universität Zürich, Winterthurerstrasse 190, CH-8057 Zürich.

e-mail:megli@geo.unizh.ch

Dr. Aldo Mirabella, Istituto Sperimentale per lo Studio e la Difesa del Suolo, Piazza M. d'Azeglio 30, I-50121 Firenze.

e-mail: aldo.mirabella@issds.it

Manuskripteingang/received/rentrée du manuscrit: 12.1.2001

Annahme zum Druck/accepted for publication/accepté à l'impression: 21.6.2001 